\title{
Chip-based wide field-of-view nanoscopy
}

Robin Diekmann ${ }^{\# 1}$, Øystein I. Helle ${ }^{\# 2}$, Cristina I. $\varnothing_{i e^{2}}$, Peter McCourt ${ }^{3}$, Thomas R. Huser $^{1,4}$, Mark Schüttpelz ${ }^{1}$ and Balpreet S. Ahluwalia ${ }^{2}$

Link to the manuscript:

https://www.nature.com/nphoton/journal/v11/n5/full/nphoton.2017.55.htmI

doi:10.1038/nphoton.2017.55 


\section{Chip-based wide field-of-view nanoscopy}

Robin Diekmann ${ }^{\# 1}$, Øystein I. Helle ${ }^{\# 2}$, Cristina I. $\varnothing_{i e^{2}}$, Peter McCourt ${ }^{3}$, Thomas R. Huser $^{1,4}$, Mark Schüttpelz ${ }^{1}$ and Balpreet S. Ahluwalia ${ }^{2}$

\# These authors contributed equally to this work.

1 Department of Physics, Bielefeld University, 33615 Bielefeld, Germany.

2 Department of Physics and Technology, UiT-The Arctic University of Norway, 9037 Tromsø, Norway.

3 Department of Medical Biology, UiT-The Arctic University of Norway, 9037 Troms $ø$, Norway.

4 Department of Internal Medicine and NSF Center for Biophotonics, University of California, Davis, Sacramento, CA

95817, USA

Correspondence should be addressed to M.S. (schuettp@physik.uni-bielefeld.de) or B.S.A.

(balpreet.singh.ahluwalia@uit.no).

Present optical nanoscopy techniques use a complex microscope for imaging and a simple glass slide to hold the sample. Here, we demonstrate the inverse: the use of a complex, but mass-producible optical chip, which hosts the sample and provides a waveguide for the illumination source, and a standard low-cost microscope to acquire super-resolved images via two different approaches. Waveguides composed of a material with high refractive-index contrast provide a strong evanescent field that is used for single-molecule switching and fluorescence excitation, thus enabling chipbased single molecule localization microscopy. Additionally, multimode interference patterns induce spatial fluorescence intensity variations that enable fluctuation-based super-resolution imaging. As chip-based nanoscopy separates the illumination and detection light paths, total-internal-reflection fluorescence excitation is possible over a large field of view, with up to $0.5 \mathrm{~mm} \times 0.5 \mathrm{~mm}$ being demonstrated. Using multicolour chip-based nanoscopy, we visualize fenestrations in liver sinusoidal endothelial cells.

Various different methods of super-resolution microscopy have emerged within the last few years ${ }^{1}$. Techniques include structured illumination microscopy $(\mathrm{SIM})^{2,3}$, stimulated emission depletion (STED) $)^{4,5}$ microscopy and temporal signal fluctuation-based techniques such as super-resolution optical fluctuation Imaging $(\mathrm{SOFI})^{6}$ and entropy based super-resolution imaging $(E S I)^{7}$. However, single molecule localization microscopy (SMLM) approaches such as (direct) stochastic optical reconstruction microscopy $((d) S T O R M)^{8,9}$ and (fluorescence) photo-activated localization microscopy (F)PALM ${ }^{10,11}$ are possibly the most widespread implementations currently used in applied nanoscopy. SMLM techniques rely on spatially and temporally separated signals from single emitters such as organic fluorophores ${ }^{9}$ or quantum $\operatorname{dots}^{12,13}$ to localize their coordinates with sub-pixel precision. The localizations coordinate table from up to multiple thousands of frames is used to render the super-resolved image, 
after possible filtering steps based on the localizations list. In contrast, fluctuation based techniques do not require strictly separated emitters but make use of temporal fluctuations of the fluorescence intensity signal over several to hundreds of frames. The super-resolved image is generated by time-dependent statistical analysis of the pixel values in the fluorescence image stack, e.g. calculation of cumulants in $\mathrm{SOFI}^{6}$ or entropy values in $\mathrm{ESI}^{7}$.

Ongoing developments suggest that an impressive diversity of biological and medical questions will be answered using optical nanoscopy. However, this development is frequently accompanied by the need for bulky and expensive optical setups that are rather cumbersome to operate and to maintain, especially for untrained users. Typically, a standard microscope is adapted by the use of bulk optical components to generate, steer and deliver the required illumination pattern to the sample Often, the same objective lens is used for both, fluorescence excitation and detection, and there are thus overlaid excitation and emission paths. Furthermore, specific objective lenses with a high numerical aperture (NA) are needed to support optical sectioning in the form of total internal reflection or highly inclined and laminated optical sheet $(\mathrm{HILO})^{14}$ illumination. Another approach to optical sectioning is given by Bessel beam ${ }^{15}$ or lattice light sheet ${ }^{16}$ illumination, again at the cost of rather complex and bulky optical setups. Besides the technical demands, using a high-NA objective lens with a high magnification consequently limits the field-of-view (FOV). This makes simultaneous super-resolution imaging of several to many cells, difficult or impossible.

Optical microscopy based on waveguide chips significantly reduces the complexity of the entire optical setup, enabling miniaturization by completely removing the excitation light path from the microscope. Instead, waveguides which tightly confine the guided light by total internal reflection due to a high refractive index contrast (HIC) to the surrounding media such as water and cells are used to deliver the illumination light to the sample. The evanescent field on top of the waveguide can be utilized for total internal reflection fluorescence (TIRF) excitation over an almost arbitrarily wide FOV that is intrinsically independent of the detection objective lens and in principle only limited by the waveguide design. Evanescent field excitation using waveguides was first introduced by Grandin et al. ${ }^{17}$, where a slab waveguide was used to generate an evanescent field over the large stretch of the waveguide chip. Slab waveguides (Fig. 1a) have later been used for both fixed and live cell imaging ${ }^{18-20}$ and recently for label free imaging ${ }^{21}$. In another work $^{22}$, a coverslip was used to support guided modes of light, providing an evanescent field across the entire coverslip. Although these previous approaches to waveguide-based illumination have been used for fluorescence microscopy for some time, none of these techniques have yet demonstrated imaging with a resolution beyond the diffraction limit. Here, we present super-resolution optical microscopy 
using chip-based waveguides made of HIC materials, opening research activities towards the field of nanoscopy based on photonic integrated circuits (PICs).

Waveguide chips potentially allow for easy integration of multiple optical functions on a common platform. Particularly, devices based on HIC materials allow tighter confinement of light inside the waveguide reducing the footprints and opening new possibilities of integrated chip-based optical systems such as Raman spectroscopy ${ }^{23}$, bio-sensors ${ }^{24}$, optical coherence tomography ${ }^{25}$, and others.

Here, we demonstrate waveguide chip-based super-resolution fluorescence imaging by two complementary approaches using ESI and dSTORM. The high intensity in the evanescent field generated by the HIC waveguide material is used for optical switching of fluorophores as required by dSTORM. In addition, the intrinsically multi-mode interference pattern within the waveguide is used to generate fluctuating intensity patterns for ESI. To demonstrate the applicability of waveguide chip-based super-resolution microscopy we visualize the connection of the actin cytoskeleton and plasma membrane fenestrations in liver sinusoidal endothelial cells (LSECs).

\section{RESULTS}

\section{Chip-based single molecule localization microscopy}

The performance of chip-based dSTORM is shown by imaging immunostained microtubules in rat LSECs ${ }^{26}$ plated directly on the waveguide (Fig. 2a). Measuring the lateral profile along one straight microtubule filament reveals a hollow structure ${ }^{27}$ which has been used earlier in localization microscopy as a benchmark sample ${ }^{28-30}$, discussed in detail $\mathrm{in}^{31}$. This shows a resolution of better than $50 \mathrm{~nm}$ (Fig. 2b), confirmed by full-width-at-half-maximum (FWHM) values, localization precision ${ }^{32}$, and Fourier ring correlation ${ }^{33,34}$ (FRC) calculations (Supplementary Fig. 1). The resolution capability was further investigated by using DNA origami nanorulers that provide markers at $(50 \pm 5) \mathrm{nm}$ distance as references. These can be clearly resolved in chip-based dSTORM (Fig. 2c,d, Supplementary Fig. 2) which shows a comparable performance to the widely used architecture of an inverted TIRF dSTORM setup (Fig. 2c,d, Supplementary Fig. 3).

As an advantage over conventional setups, waveguide chip-based nanoscopy greatly benefits from the fact that the fluorescence excitation is independent of the detection objective lens. As fluorescence is excited by the evanescent field of the waveguide, the technique provides optical sectioning and excellent signal to background ratios at penetration depths below $200 \mathrm{~nm}$ (Supplementary Fig. 4, Supplementary Fig. 5, Video 1), similar to 
objective-based TIRF. Beyond that, the planar extent of the evanescent field only depends on the waveguide geometry and can thus be adjusted by the waveguide layout. While for epiillumination, dSTORM imaging over a FOV of $100 \mu \mathrm{m}$ side lengths has recently been demonstrated $^{35}$, we significantly extend this range by chip-based TIRF imaging: the use of a broad waveguide in combination with a low magnification/low NA objective lens enables simultaneous imaging of more than 50 cells (Fig. $2 \mathbf{e}$ ) on a FOV with 0.5 millimetre width and height while details below $140 \mathrm{~nm}$ could still be resolved (Fig. 2f,g), again confirmed by multiple methods (Supplementary Fig. 6).

Samples can be prepared directly on the waveguide chip (Fig. 1b) following standard protocols used for cell attachment to common glass coverslips ${ }^{36}$. Besides acting as a substrate for the sample, the chip additionally provides an evanescent field used for fluorescence excitation through the integrated waveguide (Fig. 1c) while the fluorescent signal is captured by a conventional upright microscope. Since light guidance and shaping is integrated in the waveguide chip, the entire optical setup features relatively low complexity and only requires efficient coupling of light into the waveguide. We meet this requirement by either focusing different laser lines through an objective lens to the input facet of the waveguide or directly coupling to it from an optical fibre (Fig. 1d). As different wavelengths can be combined simultaneously, dyes can be effectively photoswitched for dSTORM imaging (Supplementary Fig. 7).

To develop suitable waveguides for chip-based nanoscopy, tantalum pentoxide $\left(\mathrm{Ta}_{2} \mathrm{O}_{5}\right)$ and silicon nitride $\left(\mathrm{Si}_{3} \mathrm{~N}_{4}\right)$ materials were used because of their high refractive indices, low propagation losses, low absorption, and low auto-fluorescence at visible wavelengths ${ }^{37}$. High intensities in the evanescent field induce switching of organic fluorophores. To achieve this, we use channel-like waveguides of rib or strip geometry (Fig. 1a) that transversely confine the guided light in contrast to the slab geometry used in other waveguide-imaging approaches ${ }^{17-19,21}$. Channel breadths (i.e. the waveguide extend orthogonal to the wave propagation direction) range from 25 to $500 \mu \mathrm{m}$ in order to span the appropriate length scale for imaging many samples simultaneously, e.g. several cells.

The waveguides used in this work are highly multi-moded. Back reflections and multi-mode interference inside the waveguide cause a spatially stable distribution of laterally non-uniform evanescent fields, which strongly depends on the coupling into the waveguide. Accordingly, fluorescence excitation is inhomogeneous and, hence, impairs the reconstructed dSTORM image. To counteract this problem, a piezo stage is used to oscillate the coupling objective lens or fibre back and forth along the input facet of the waveguide during the measurement. 
This maintains continuous coupling but shifts the mode pattern to obtain an average distribution which shows significantly less modulation over larger length scales as compared to conventional TIRF setups (Supplementary Fig. 8, Supplementary Fig. 9, Video 2). Consequently, the distinct modes are not visible in the reconstructed $d S T O R M$ images.

\section{Chip-based fluctuation imaging}

In contrast, fluctuations induced by the shifted mode pattern are desired in the case of chipbased ESI. Although original implementations of the fluctuation-based approaches SOFI and ESI use intrinsic quantum dot or fluorophore temporal intensity fluctuations (e.g. due to blinking and bleaching), it has recently been shown that speckle pattern illumination ${ }^{38}$ can also invoke temporal emission fluctuations allowing for super-resolved fluctuation imaging ${ }^{39}$. Intrinsic intensity fluctuations originate from single emitters and therefore spatially tightly confined sources. On the contrary, the spatial frequencies of the waveguide illumination pattern define the length scales on which the fluctuations occur, and, hence, the obtainable resolution (Supplementary Fig. 10, Supplementary Note 1). Using dSTORM, we have measured fringes in the multi-mode interference pattern of a waveguide. The FWHM of the structure sizes goes down to $140 \mathrm{~nm}$ for a vacuum laser excitation wavelength of $660 \mathrm{~nm}$ (Supplementary Fig. 11).

To utilize the illumination intensity fluctuations induced by the waveguide for ESI analysis, we oscillate the coupling along the input facet such that the mode distribution changes from image to image. As random intensity fluctuations are desired, there is no need to further control the illumination pattern besides changing it from frame to frame. Low input power is used, keeping the intensity under the threshold of undesired single molecule switching, contrary to the dSTORM acquisition procedure where single molecule switching is required. While continuously changing the excitation pattern in this manner, about 200 frames are recorded. The acquired data is used as input for the ESI reconstruction algorithm to generate a super-resolved image (Fig. 3a) which we demonstrate via imaging tubulin in LSECs (Fig. $3 b, c)$.

Following the ESI acquisition, the same sample is again imaged using dSTORM by increasing the illumination intensity. This allows for a direct comparison of the ESI image with the dSTORM image of higher resolution but requiring a much higher number of input frames (Supplementary Table 1). Hence, dSTORM serves as a control reference for assessing the performance of chip-based ESI. This verifies that the resolution of the ESI image is on the order of $110 \mathrm{~nm}$, as adjacent microtubules at $106 \mathrm{~nm}$ distance are still resolved and simultaneously observed in both super-resolved images (Fig. 3c,d). This resolution is also 
confirmed by a FWHM of $104 \mathrm{~nm}$ of a single tubule in the ESI image (Supplementary Fig. 12). Thus, chip-based ESI utilizing spatial excitation pattern fluctuations readily achieves a resolution enhancement of about a factor of 2, using an NA 1.2 objective lens for fluorescence detection (Supplementary Note 1). Chip-based dSTORM pushes the resolution even further, admittedly at the cost of longer acquisition times.

\section{Scalable super-resolution imaging}

As the evanescent field generation in waveguide chip-based nanoscopy does not depend on the objective lens used for fluorescence detection, the presented approaches can be applied for successive image acquisitions at different magnifications allowing for scalable FOV imaging: To obtain an overview image with a large FOV, low magnification/ low NA lenses can be used. If higher resolution is desired, specific regions of interest (ROI) can be imaged at superior resolution afterwards switching to a high magnification/ high NA objective lens.

Intensity fluctuations owing to the shifted mode pattern enabled us to record ESI images with TIRF excitation in a FOV of $0.46 \mathrm{~mm}$ width using a 20x magnification/ NA 0.4 objective lens (Figure 4b,e). While a resolution not better than $653 \mathrm{~nm}$ is expected for diffraction-limited imaging (Fig. 4a,d), actin filaments of $334 \mathrm{~nm}$ distance can still be resolved in the large FOV ESI image (Fig. 4h). A filament FWHM value of $333 \mathrm{~nm}$ confirms this finding (Supplementary Fig. 13). This demonstrates a resolution enhancement at these particular positions of approx. a factor of 2, which was achieved from 208 frames recorded in less than 25 seconds. For further resolution improvement, dSTORM imaging was performed using the same low NA objective lens (Fig. 4c,f).

The use of a low NA 0.45 (Fig. 2e,f) or 0.4 (Fig. 4c,f) objective lens lacks resolution compared to the use of a higher NA 1.2 objective lens (Fig. 2a, Fig 3b,c, Fig. $\mathbf{4 g}$ ) as the obtainable localization precision and accuracy strongly depends on the NA of the utilized objective lens (Supplementary Note 2, Supplementary Note 3, Supplementary Fig. 14). Nevertheless, it shows that TIRF-based microscopy is not limited to high NA and high magnification lenses and can be extended to wide fields-of-view. Using the low NA 0.4 objective lens, as few as 208 frames for ESI and 3,000 frames for dSTORM were sufficient to capture the structure of interest at significantly improved resolution in comparison to the diffraction limit (Supplementary Note 4, Supplementary Fig. 15, Supplementary Fig. 16). Fast software tools for (almost) real-time dSTORM reconstruction are readily available ${ }^{40,41}$, such that $d S T O R M$ is mainly limited by the acquisition time. Hence, either low NA ESI or dSTORM can be used for identification of cells of interest or for finding rare biological events, while the choice of the proper method can be made by prioritizing either the acquisition time 
(choosing ESI) or the resolution (choosing dSTORM). Subsequently, an identified region of interest may be imaged using high NA dSTORM to achieve uncompromised resolution (Fig. 4g). Additionally, the relatively short acquisition times for the low NA images help to prevent photobleaching of fluorophores, allowing for successive acquisition procedures of the same sample (Supplementary Fig. 15).

\section{Imaging membrane/cytoskeleton interaction in LSECs}

Besides the cytoskeleton, the plasma membrane of LSECs is of distinct importance for their function $^{26,42}$. LSECs are highly specialized endothelial cells forming the sinusoidal blood vessels in the liver. They contain multiple trans-cytoplasmic pores (fenestrations) of 50 to $200 \mathrm{~nm}$ in diameter, making optical nanoscopy a particularly well-suited tool for their investigation ${ }^{43}$. Fenestrations are typically grouped together in structures called sieve plates, due to their function as a molecular sieve between the blood and the hepatocytes. Fenestrations allow free passage of plasma from the sinusoidal lumen through to the Space of Disse and beyond to the underlying hepatocytes (and in the reverse direction), while retaining the blood cells in the lumen. Constituents in the plasma (e.g. lipoproteins, pharmaceuticals) can thus be cleared and processed by hepatocytes, and products synthesized by hepatocytes can be released back into the circulation via this route. We applied waveguide imaging to visualize the interaction between the actin network and fenestrations (Fig. 5a, Supplementary Fig. 17). Multi-colour chip-based dSTORM shows that small actin filaments colocalize with the membrane supporting the individual fenestrations (Fig. 5b), reinforcing earlier findings with different methods ${ }^{44,45}$. Thicker actin bundles surround multiple fenestrations forming sieve plates. Recently, it has been demonstrated that sieve plates are also surrounded by tubulin ${ }^{44}$. Taken together with our results, this suggests that sieve plates have a combined actin and tubulin outer framework. Super-resolution optical methods that allow multiplexing will contribute to a greater understanding of how sieve plates and fenestrations function and are regulated, especially if these methods can be extended to living cells.

\section{DISCUSSION}

Our approach to resolution-scalable nanoscopy allows for localization-based, as well as fluctuation-based super-resolution imaging on a simple setup that only demands coupling of the excitation light source into the waveguide. Using a 20x objective lens, a FOV of approx. $0.5 \mathrm{~mm}$ width can be imaged at a resolution of about $340 \mathrm{~nm}$ in less than 25 seconds as shown above, which will also enable live-cell applications. Accepting longer acquisition times of approx. 8 to 30 minutes, a resolution even below $140 \mathrm{~nm}$ can be achieved for this large FOV without image stitching (Supplementary Note 5). Thus, our waveguide chip-based 
approach fills the missing gap of acquiring highly resolved images over an unprecedented large FOV in TIRF microscopy of which the maximum breadth is predetermined by the waveguide layout. Changing to a $60 x$ objective lens with NA 1.2 results in an optical resolution of below $50 \mathrm{~nm}$ for dSTORM or about $110 \mathrm{~nm}$ for ESI. Though the presented approach is more flexible in the choice of the imaging lens than other implementations of TIRF microscopy, it shares the feature of a shallow sample illumination. Thus, fluorescence excitation is limited to a few hundred nanometres above the substrate, which can be either advantageous or disadvantageous depending on the application. While the illumination over the entire stretch of the waveguide enables large FOV TIRF imaging, the illuminated area usually goes beyond the FOV. All samples located on top of the currently imaged waveguide are simultaneously exposed to fluorescence excitation which must be considered in the experimental design, e.g. concerning photobleaching.

Though both ESI and dSTORM are based on fluorescence signal dynamics, the underlying processes significantly differ for their chip-based implementations. As in conventional realisations, chip-based dSTORM relies on the ability of the dyes to exhibit photoswitching, but the demonstrated implementation of ESI induces spatial intensity fluctuations by the waveguide excitation. It is therefore not restricted to specific probes, which makes it applicable to an even wider range of scenarios. So far, we have focused on a comparison of ESI and dSTORM, which on the one hand allowed us to validate chip-based ESI by dSTORM. On the other hand, experiments were designed for dSTORM compatible dyes and imaging was performed in a buffer that favours photoswitching beyond a certain intensity threshold. As photoswitching was not desired for ESI data acquisition, excitation intensities were kept relatively low, entailing the need for rather long camera exposure times. However, if chip-based ESI is performed independently of dSTORM, much higher intensities accompanied with significantly shorter exposure times can be applied. This will enable the acquisition of a fully reconstructed ESI image within 1 second, allowing for live-cell imaging at high temporal resolution.

While dSTORM and fluctuation-based approaches already belong to the most wide-spread techniques for super-resolution imaging, the waveguide chip-based implementation dramatically reduces the complexity of the setup and, thus, has the potential to make it accessible for a wider range of users. Incorporating it into PICs will make it compatible with fast and compact optical fibre components, e.g. fibre-based switches, modulators, multiplexer and lasers. A waveguide chip, either fibre pigtailed or with a FC connector, will simply allow adding or removing any optical fibre components, thus eliminating the need of system re-alignment which is often the case when using bulk optical components. By guiding 
the illumination light through optical fibres and waveguides, many standard optical microscopes can be used to acquire super-resolved images. Future PIC developments towards on-chip laser generation, filtering, and steering of entire illumination systems will potentially further extend the capabilities. Additionally, the integrated platform makes combinations with different lab-on-a-chip methods, e.g. microfluidics ${ }^{46}$, optical trapping ${ }^{47}$ or other detection techniques ${ }^{48}$ now straightforward to implement. Furthermore, other analysis modes based on SMLM can be utilized e.g. for highly sensitive quantitative binding assays ${ }^{49}$. 


\section{METHODS}

Methods and any associated references are available in the online version of the paper.

\section{ACKNOWLEDGMENTS}

The authors thank Idir Yahiatène and Marcel Müller for help with the ESI reconstruction algorithm and Viola Mönkemöller for help with sample preparation and membrane dye staining. This work was supported by the European Research Council (grant no. 336716 to B.S.A.), the Research Council of Norway (grant no. 244764/F11 to B.S.A.), and the German Academic Exchange Service (grant no. 57160327 to M.S.). R.D. acknowledges additional support by grant no. KF2140610NT4 of the German Federal Ministry for Economic Affairs and Energy.

\section{AUTHOR CONTRIBUTIONS}

B.S.A. and M.S. conceived the project. All authors designed the research. C.I.Ø. isolated the cells, and stained and prepared the biological samples. R.D. and Ø.I.H. built the setup, prepared the non-biological samples, performed the experiments, performed the simulations, reconstructed the images, analysed the data, and created the figures. R.D., Ø.I.H., M.S., and B.S.A. mainly wrote the paper. All authors reviewed the manuscript.

\section{COMPETING FINANCIAL INTERESTS}

M.S. and B.A.S. applied for patent GB1606268.9 for chip-based optical nanoscopy. The other authors declare no competing financial interest. 
Figure 1 | The implementation of chip-based nanoscopy. (a) Channel-like waveguide geometries are realized by etching the slab waveguide either partially or completely down to the $\mathrm{SiO}_{2}$ substrate. In either case, the light is mainly guided by the channels of 25 to $500 \mu \mathrm{m}$ breadth. (b) Five channels can easily be seen in the photograph of a strip waveguide chip, marked by the arrows. (Scale bar, $1 \mathrm{~cm}$.) (c) Light guided inside the waveguide is the source of the evanescent field illuminating samples on top of the surface. (d) The optical setup consists of a simple upright microscope for fluorescence detection and an illumination unit to provide coupling to the input facet of the waveguides either through an objective lens or via a lensed fibre.

a

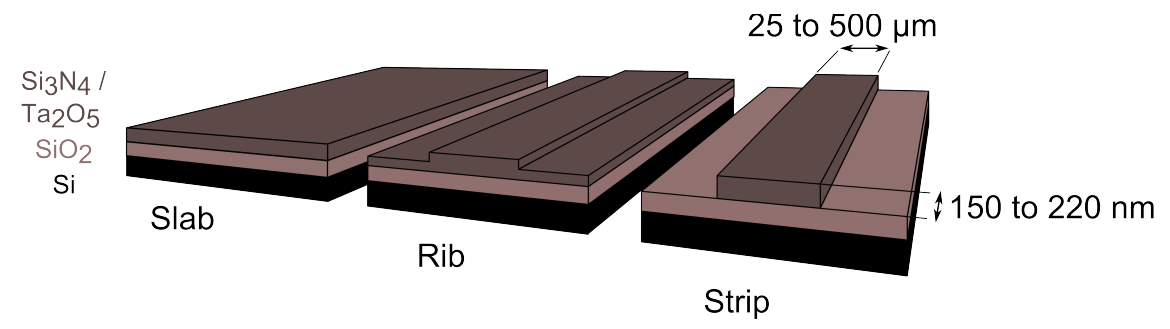

C

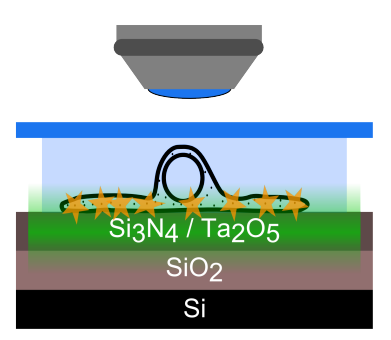

d

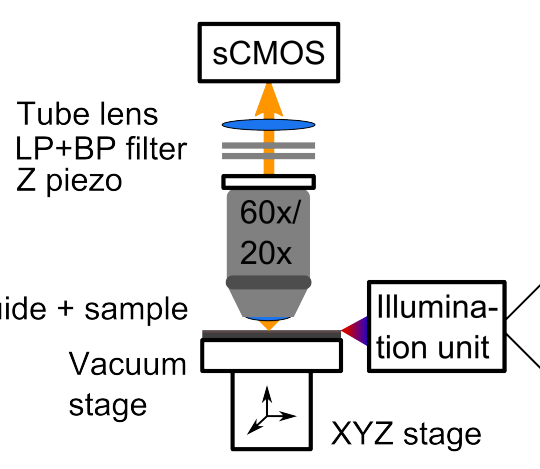

Waveguide + sample b

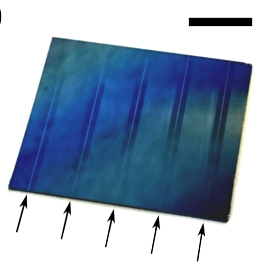

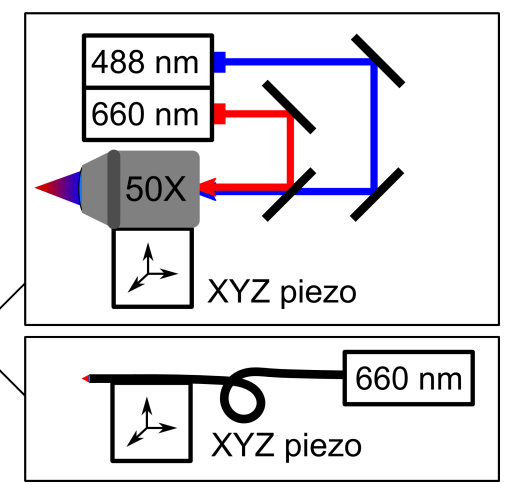


Figure 2 | Demonstration of chip-based dSTORM. (a) Diffraction limited and dSTORM imaging of tubulin in liver sinusoidal endothelial cells (LSECs). (Scale bar, $2 \mu \mathrm{m}$. Scale bar on inset, $1 \mu \mathrm{m}$.) Measuring a lateral profile of $540 \mathrm{~nm}$ width along a straight microtubule (magenta marking in the inset of the $d S T O R M$ image) reveals its hollow structure (b). (c) The resolution capability is further investigated by imaging DNA-origami nanorulers of $(50 \pm 5) \mathrm{nm}$ specified length that can be clearly resolved with waveguide-based $d S T O R M$ similar to objective-based TIRF dSTORM. (Scale bars, $50 \mathrm{~nm}$.) (d) Analysing their line profiles, a mean nanoruler length of $49 \mathrm{~nm}$ is found in both cases affirming that the chip-based implementation shows comparable performance to a conventional inverted dSTORM setup. (e) Additionally, waveguide chip-based illumination allows for using a low magnification/ low NA (20x/ NA 0.45) objective lens for dSTORM imaging over a field-of-view of $0.5 \mathrm{~mm} \times 0.5$ $\mathrm{mm}$. (f) shows a detail marked by the white box in (e), where the profile over adjacent tubulin filaments reveals their separation by $138 \mathrm{~nm}(\mathrm{~g})$.

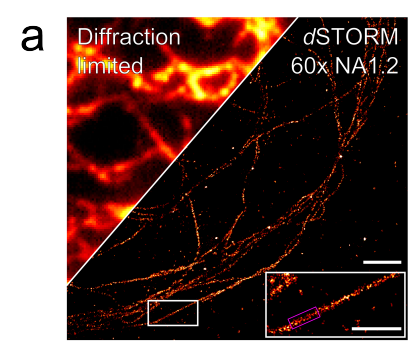

C Objective-based Waveguide chipC TIRF dSTORM based dSTORM

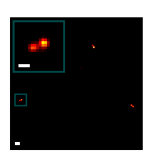

d

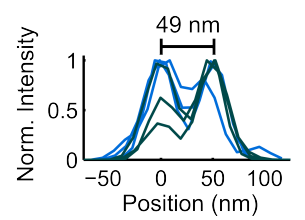

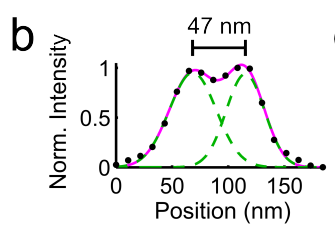
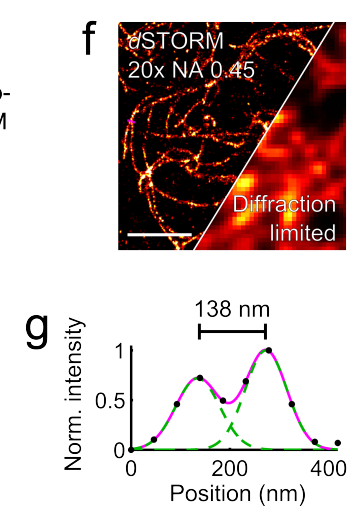

e

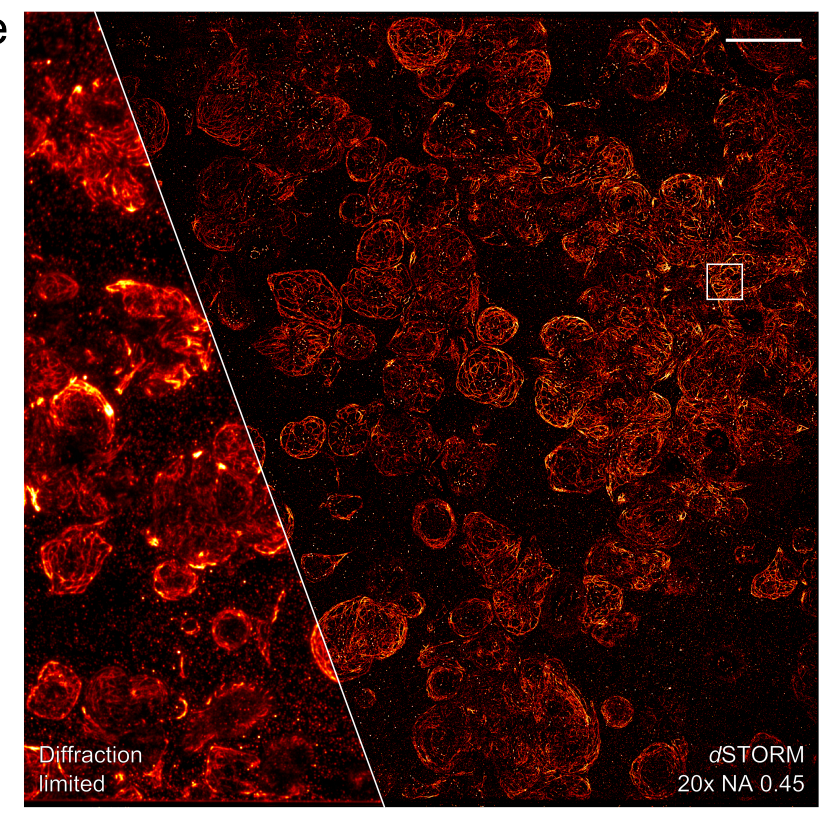


Figure 3 | Demonstration of chip-based ESI. (a) Spatial fluorescence intensity fluctuations are induced by changing the mode pattern of the waveguide during image acquisition. Accordingly, these measurements show a diffraction limited image of the labelled structure multiplied by the mode pattern. A stack of approx. 200 frames is used as input data for the fluctuation analysis reconstruction algorithm, resulting in one super-resolved image. (b) Imaging tubulin in an LSEC. The comparison of the diffraction limited image, the corresponding ESI reconstruction, and the dSTORM image shows the gradually increasing resolution. (Scale bar, $5 \mu \mathrm{m}$.) (c) shows a magnification of the same region (indicated by the rectangle in (b)) for the three different imaging modalities. (Scale bar, $0.5 \mu \mathrm{m}$.) The dSTORM image of the same structure verifies the applicability of chip-based ESI: the line profiles reveal a resolution of $106 \mathrm{~nm}$ for chip-based ESI (green line) by imaging two adjacent microtubules, simultaneously observed in the dSTORM image (blue dashed line) (d).

Shifted excitation pattern:

intensity fluctuations

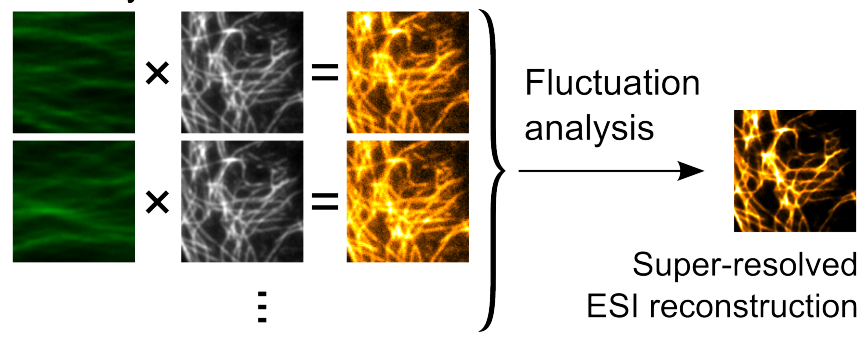

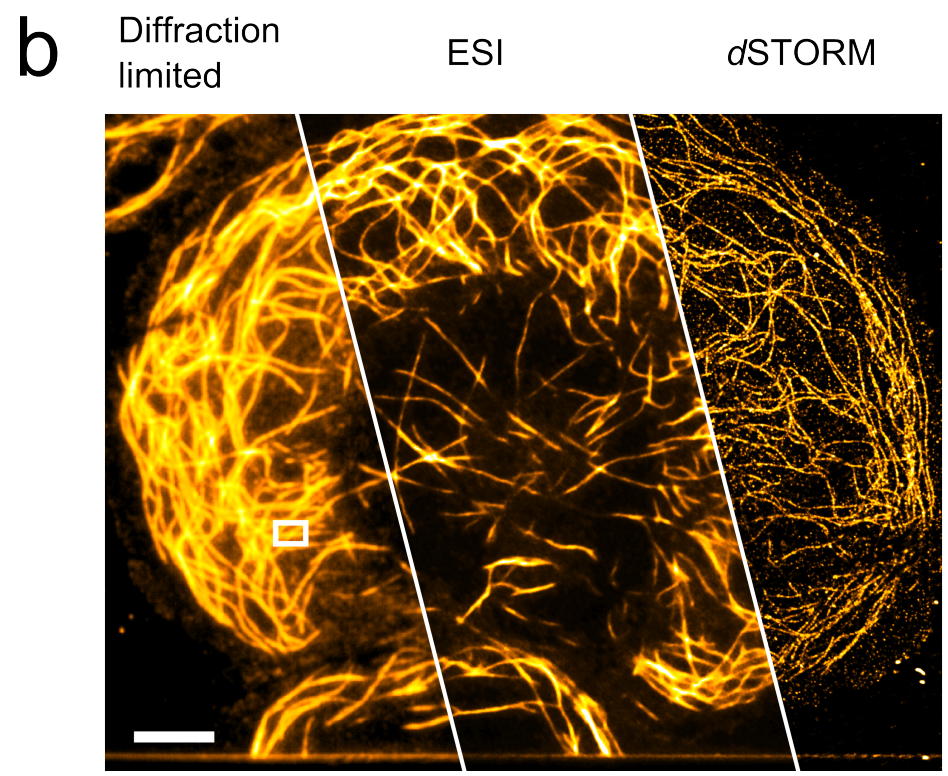
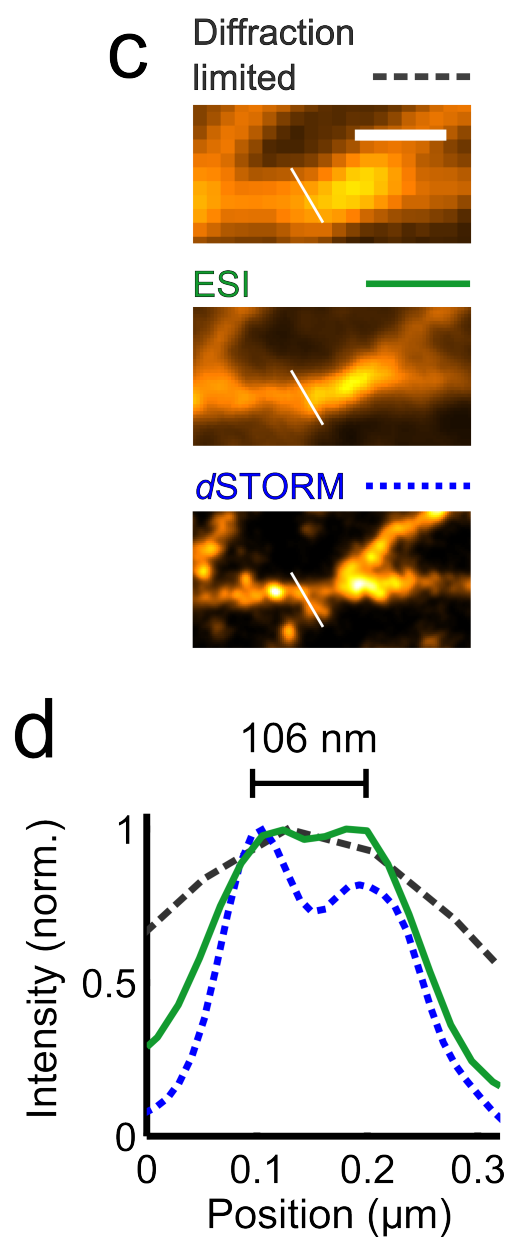
Figure 4 | Imaging the same sample under varied acquisition conditions reveals the specific strengths of the different approaches. As TIRF excitation is provided over the entire width of the waveguide, arbitrary objective lenses can be used for detection. Using a 20x magnification objective lens allows for chip-based TIRF imaging over a field-of-view of $0.46 \mathrm{~mm}$ width (a,d). Both fluctuation-based ESI (b,e) and localization-based dSTORM (c,f) result in an optical resolution enhancement obtained using the NA 0.4 lens, and, thus, resolve actin bundles at $334 \mathrm{~nm}$ distance (h). Hence, both techniques provide a tool for scanning large fields-of-view at high resolution to identify cells of interest that can be reimaged using a high magnification/ high NA lens in a subsequent step (g), accomplishing even superior resolution. The choice between wide field-of-view ESI or dSTORM can be made on either prioritizing short acquisition times (choosing $\mathrm{ESI}$ ) or best resolution (choosing dSTORM). (d-g) show a detail of the region marked by the box in (a). (Scale bar on (a), 20 $\mu \mathrm{m}$. Scale bar on (d), $2 \mu \mathrm{m}$.)
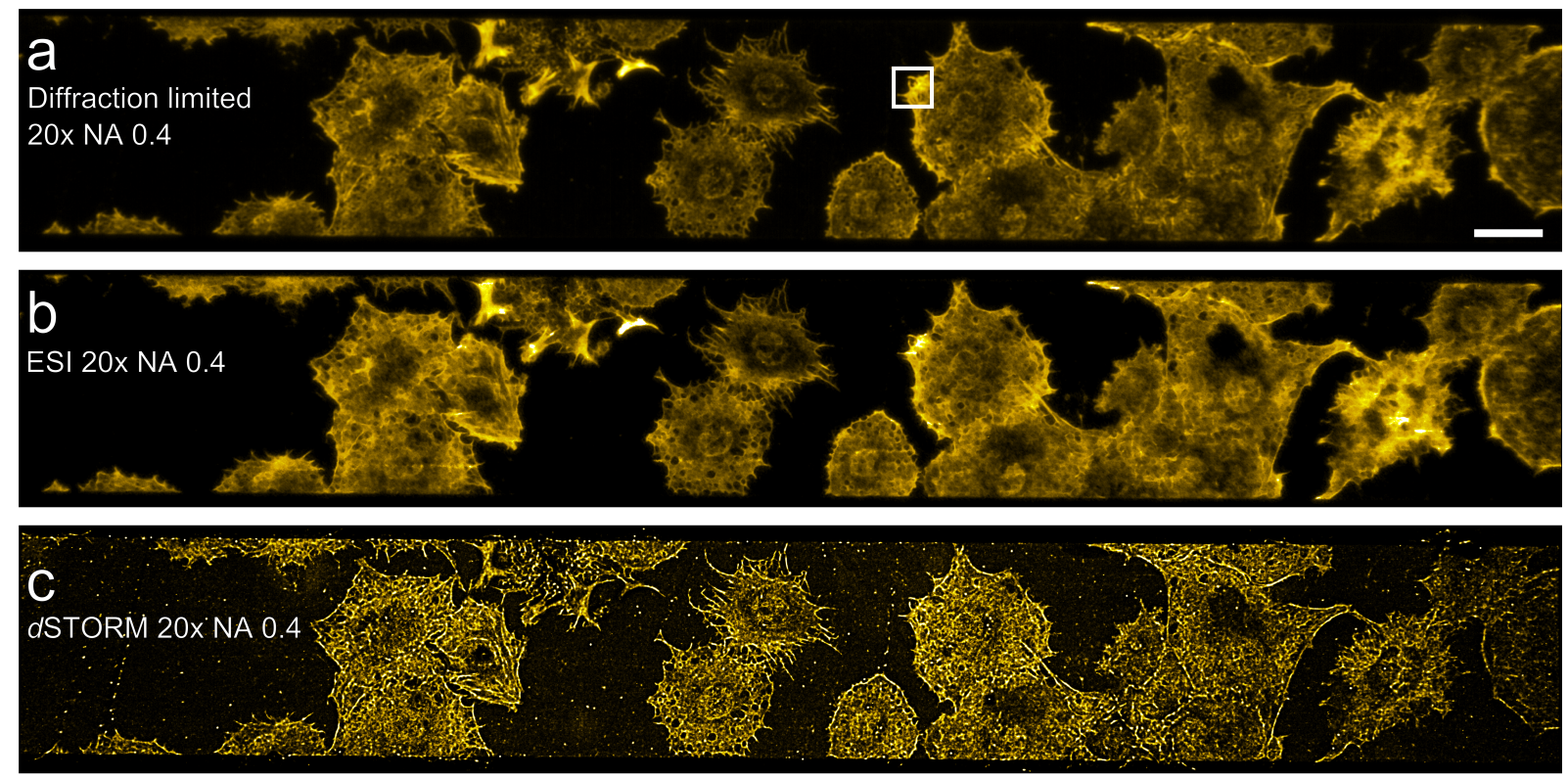

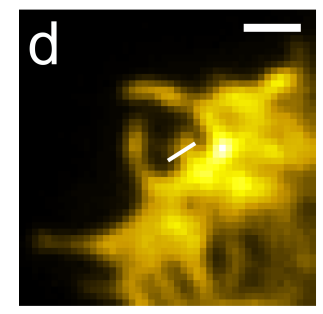

Diffraction limited $----$

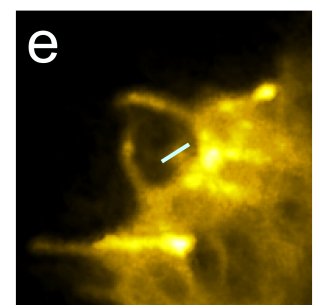

ESI 20x NA 0.4 -......

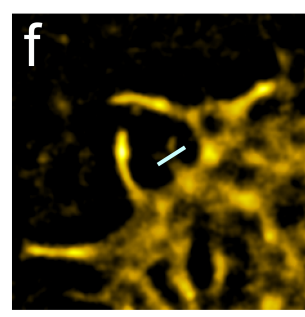

dSTORM 20x NA 0.4

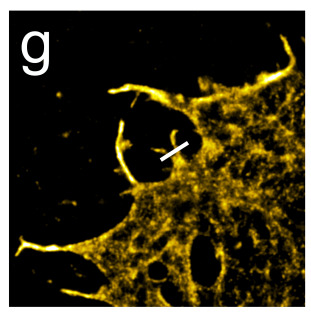

dSTORM 60x NA 1.2

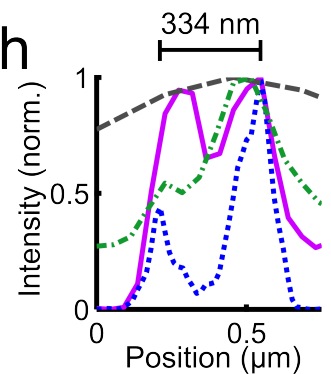


Figure 5 | Multi-colour chip-based dSTORM reveals the interplay between actin (magenta) and the membrane (green) in LSECs. (a) Groups of fenestrations form sieve plate superstructures which are surrounded by thicker actin bundles. As can be seen in the inset, actin is present between neighbouring fenestrations where it colocalizes with the plasma membrane. (Scale bar, $5 \mu \mathrm{m}$. Scale bar on inset, $1 \mu \mathrm{m}$.) (b) The line profiles taken at different positions in the liver cell as shown in (a) reveal diameters of approx. $200 \mathrm{~nm}$ and smaller for the fenestrations chosen, that can be resolved by super-resolution microscopy only. The profiles underline the visual impressions of colocalization.
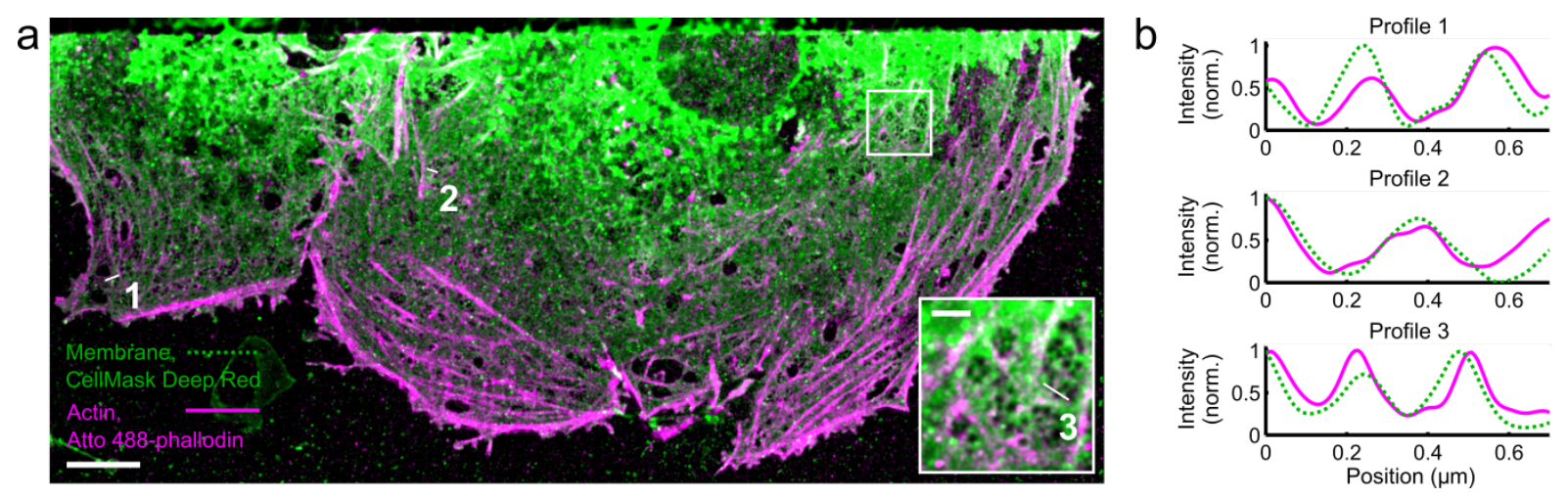


\section{REFERENCES}

1 Schermelleh, L., Heintzmann, R. \& Leonhardt, H. A guide to super-resolution fluorescence microscopy. Journal of Cell Biology 190, 165-175, doi:10.1083/jcb.201002018 (2010).

2 Gustafsson, M. G. Surpassing the lateral resolution limit by a factor of two using structured illumination microscopy. J Microsc 198, 82-87 (2000).

3 Heintzmann, R. \& Cremer, C. G. Laterally modulated excitation microscopy: improvement of resolution by using a diffraction grating. Proc. SPIE 3568, Optical Biopsies and Microscopic Techniques III 3568, 185-196, doi:10.1117/12.336833 (1999).

4 Hell, S. W. \& Wichmann, J. Breaking the diffraction resolution limit by stimulated emission: stimulated-emission-depletion fluorescence microscopy. Opt Lett 19, 780782 (1994).

$5 \quad$ Willig, K. I., Rizzoli, S. O., Westphal, V., Jahn, R. \& Hell, S. W. STED microscopy reveals that synaptotagmin remains clustered after synaptic vesicle exocytosis. Nature 440, 935-939, doi:10.1038/nature04592 (2006).

6 Dertinger, T., Colyer, R., lyer, G., Weiss, S. \& Enderlein, J. Fast, background-free, 3D super-resolution optical fluctuation imaging (SOFI). Proc Natl Acad Sci U S A 106, 22287-22292, doi:10.1073/pnas.0907866106 (2009).

7 Yahiatene, I., Hennig, S., Muller, M. \& Huser, T. Entropy-Based Super-Resolution Imaging (ESI): From Disorder to Fine Detail. Acs Photonics 2, 1049-1056, doi:10.1021/acsphotonics.5b00307 (2015).

8 Rust, M. J., Bates, M. \& Zhuang, X. Stochastic optical reconstruction microscopy (STORM) provides sub-diffraction-limit image resolution. Nature Methods 3, 793-795 (2006).

9 Heilemann, M. et al. Subdiffraction-resolution fluorescence imaging with conventional fluorescent probes. Angew Chem Int Edit 47, 6172-6176, doi:10.1002/anie.200802376 (2008).

10 Betzig, E. et al. Imaging intracellular fluorescent proteins at nanometer resolution. Science 313, 1642-1645, doi:10.1126/science.1127344 (2006).

11 Hess, S. T., Girirajan, T. P. K. \& Mason, M. D. Ultra-high resolution imaging by fluorescence photoactivation localization microscopy. Biophys J 91, 4258-4272, doi:10.1529/biophysj.106.091116 (2006).

12 Hoyer, P., Staudt, T., Engelhardt, J. \& Hell, S. W. Quantum dot blueing and blinking enables fluorescent microscopy. Nano Lett 11, 245-250 (2010).

13 Xu, J. Q., Tehrani, K. F. \& Kner, P. Multicolor 3D Super-resolution Imaging by Quantum Dot Stochastic Optical Reconstruction Microscopy. Acs Nano 9, 2917-2925, doi:10.1021/nn506952g (2015).

14 Makio Tokunaga, N. I., Kumiko Sakata-Sogawa. Highly inclined thin illumination enables clear single-molecule imaging in cells. Nature Methods 5, 159-161 (2007).

15 Planchon, T. A. et al. Rapid three-dimensional isotropic imaging of living cells using Bessel beam plane illumination. Nature Methods 8, 417-U468, doi:10.1038/Nmeth.1586 (2011).

16 Chen, B. C. et al. Lattice light-sheet microscopy: Imaging molecules to embryos at high spatiotemporal resolution. Science 346, 439-+, doi:10.1126/Science.1257998 (2014).

17 Grandin, H. M., Stadler, B., Textor, M. \& Voros, J. Waveguide excitation fluorescence microscopy: A new tool for sensing and imaging the biointerface. Biosens Bioelectron 21, 1476-1482, doi:10.1016/j.bios.2005.06.011 (2006).

18 Agnarsson, B., Ingthorsson, S., Gudjonsson, T. \& Leosson, K. Evanescent-wave fluorescence microscopy using symmetric planar waveguides. Opt Express 17, 50755082, doi:10.1364/Oe.17.005075 (2009). 
19 Agnarsson, B., Jonsdottir, A. B., Arnfinnsdottir, N. B. \& Leosson, K. On-chip modulation of evanescent illumination and live-cell imaging with polymer waveguides. Opt Express 19, 22929-22935, doi:10.1364/OE.19.022929 (2011).

20 Shen, H. et al. TIRF microscopy with ultra-short penetration depth. Opt Express 22, 10728-10734, doi:10.1364/OE.22.010728 (2014).

21 Agnarsson, B. et al. Evanescent Light-Scattering Microscopy for Label-Free Interfacial Imaging: From Single Sub-100 nm Vesicles to Live Cells. ACS Nano 9, 11849-11862, doi:10.1021/acsnano.5b04168 (2015).

22 Ramachandran, S., Cohen, D. A., Quist, A. P. \& Lal, R. High performance, LED powered, waveguide based total internal reflection microscopy. Scientific reports 3 (2013).

23 Dhakal, A. et al. Evanescent excitation and collection of spontaneous Raman spectra using silicon nitride nanophotonic waveguides. Optics Letters 39, 4025-4028, doi:10.1364/OI.39.004025 (2014).

24 Fedyanin, D. Y. \& Stebunov, Y. V. All-nanophotonic NEMS biosensor on a chip. Scientific Reports 5, doi:10.1038/srep10968 (2015).

25 Yurtsever, G. et al. Photonic integrated Mach-Zehnder interferometer with an on-chip reference arm for optical coherence tomography. Biomed Opt Express 5, 1050-1061, doi:10.1364/BOE.5.001050 (2014).

26 Sørensen, K. K., Simon-Santamaria, J., McCuskey, R. S. \& Smedsrød, B. Liver Sinusoidal Endothelial Cells. Comprehensive Physiology (2015).

27 Weber, K., Rathke, P. C. \& Osborn, M. Cytoplasmic microtubular images in glutaraldehyde-fixed tissue culture cells by electron microscopy and by immunofluorescence microscopy. Proc Natl Acad Sci U S A 75, 1820-1824 (1978).

28 Olivier, N., Keller, D., Gonczy, P. \& Manley, S. Resolution Doubling in 3D-STORM Imaging through Improved Buffers. Plos One 8, doi:10.1371/journal.pone.0069004 (2013).

29 Vaughan, J. C., Jia, S. \& Zhuang, X. Ultra-bright Photoactivatable Fluorophores Created by Reductive Caging. Nat Methods 9, 1181-1184, doi:10.1038/nmeth.2214PMCID: PMC3561463 (2012).

30 Endesfelder, U. \& Heilemann, M. Art and artifacts in single-molecule localization microscopy: beyond attractive images. Nat Methods 11, 235-238, doi:10.1038/nmeth.2852 (2014).

31 Bourg, N. et al. Direct optical nanoscopy with axially localized detection. Nature Photonics 9, 587-593, doi:10.1038/nphoton.2015.132 (2015).

32 Endesfelder, U., Malkusch, S., Fricke, F. \& Heilemann, M. A simple method to estimate the average localization precision of a single-molecule localization microscopy experiment. Histochemistry and cell biology 141, 629-638 (2014).

33 Nieuwenhuizen, R. P. et al. Measuring image resolution in optical nanoscopy. Nat Methods 10, 557-562, doi:10.1038/nmeth.2448 (2013).

34 Banterle, N., Bui, K. H., Lemke, E. A. \& Beck, M. Fourier Ring Correlation as a resolution criterion fer super resolution microscopy. Journal of Structural Biology 183 (2013).

35 Douglass, K. M., Sieben, C., Archetti, A., Lambert, A. \& Manley, S. Super-resolution imaging of multiple cells by optimised flat-field epi-illumination. Nat Photonics 10 , 705-708, doi:10.1038/nphoton.2016.200 (2016).

36 Smedsrød, B. \& Pertoft, H. Preparation of pure hepatocytes and reticuloendothelial cells in high yield from a single rat liver by means of Percoll centrifugation and selective adherence. Journal of leukocyte biology 38, 213-230 (1985).

37 Ahluwalia, B. S. et al. Fabrication of submicrometer high refractive index Tantalum Pentoxide waveguides for optical propulsion of microparticles. Photonics Technology Letters, IEEE 21, 1408-1410 (2009).

38 Ventalon, C. \& Mertz, J. Quasi-confocal fluorescence sectioning with dynamic speckle illumination. Optics Letters 30, 3350-3352, doi:Doi 10.1364/OI.30.003350 (2005). 
39 Kim, M., Park, C., Rodriguez, C., Park, Y. \& Cho, Y. H. Superresolution imaging with optical fluctuation using speckle patterns illumination. Sci Rep-Uk 5, 16525, doi:10.1038/srep16525 (2015).

40 Wolter, S. et al. rapidSTORM: accurate, fast open-source software for localization microscopy. Nature methods 9, 1040-1041 (2012).

41 Smith, C. S., Joseph, N., Rieger, B. \& Lidke, K. A. Fast, single-molecule localization that achieves theoretically minimum uncertainty. Nature methods 7, 373-375 (2010).

42 Cogger, V. C., Roessner, U., Warren, A., Fraser, R. \& Le Couteur, D. G. A Sieve-Raft Hypothesis for the regulation of endothelial fenestrations. Computational and structural biotechnology journal 8, 1-9 (2013).

43 Mönkemöller, V. et al. Imaging fenestrations in liver sinusoidal endothelial cells by optical localization microscopy. Phys Chem Chem Phys 16, 12576-12581, doi:10.1039/c4cp01574f (2014).

44 Mönkemöller, V., Øie, C., Hübner, W., Huser, T. \& McCourt, P. Multimodal superresolution optical microscopy visualizes the close connection between membrane and the cytoskeleton in liver sinusoidal endothelial cell fenestrations. Scientific reports $\mathbf{5}$ (2015).

45 Braet, F. \& Wisse, E. Structural and functional aspects of liver sinusoidal endothelial cell fenestrae: a review. Comp Hepatol 1, 1 (2002).

46 Wang, X. et al. Enhanced cell sorting and manipulation with combined optical tweezer and microfluidic chip technologies. Lab on a Chip 11, 3656-3662 (2011).

47 Helle, Ø. I., Ahluwalia, B. S. \& Hellesø, O. G. Optical transport, lifting and trapping of micro-particles by planar waveguides. Opt Express 23, 6601-6612 (2015).

48 Dullo, F. T. \& Helles $\varnothing$, O. G. On-chip phase measurement for microparticles trapped on a waveguide. Lab on a Chip 15, 3918-3924 (2015).

49 Jain, A. et al. Probing cellular protein complexes using single-molecule pull-down. Nature 473, 484-U322, doi:10.1038/nature10016 (2011). 


\section{METHODS}

\section{Optical setups.}

Waveguide-based imaging was performed on a conventional upright microscope (BXFM, Olympus), retrofitted with a waveguide chip module consisting of a piezo stage (17MAX604, Melles Griot) holding an objective lens (LMPlanFI 50x/0.5 BD, Olympus) or a lensed fibre (HP460sm, WT\&T) used for coupling into the waveguide. The piezo allows fine tuning of the coupling, and was also programed through a piezo controller (BPC303, Thorlabs) to oscillate during image acquisition. The chip was held by a vacuum chuck on a translation stage (MBT402D, Thorlabs) to prevent movement during coupling. During dSTORM image acquisition, the vacuum pump was turned off to minimize sample drift but suction was retained. Imaging was performed using a 20x NA 0.4 (Plan N 20x/0.4, Olympus), 20x NA 0.45 (LUCPlanFL N 20x/0.45, Olympus), a 60x NA 1.2 water immersion (UplanSApo 60x/1.2 w, Olympus), or a 60x NA 1.42 oil immersion (PlanApo N 60x/1.42 oil, Olympus) objective lens. Different emission filter sets of both LP and BP filters were used (488LP (AHF) and 520/36BP (Edmund), or 664LP (AHF) and 692/40BP (AHF)), and the signal was captured by a sCMOS camera (Hamamatsu Orca Flash 4 v2), controlled by the manufacturer software (Hamamatsu HClmage). The backprojected pixel width and height was $75.9 \mathrm{~nm}$ for Atto 488 and CellMask Deep Red measurements and 108.33 nm for Alexa 647 measurements.

Illumination was provided to the chip either via coupling a laser into the lensed fibre or by combining several laser lines through the coupling objective lens. Consequently, multi-colour imaging could be performed simultaneously if the emission path was split into two spectrally separated channels. The illumination unit consisted of two solid state lasers (Oxxius LBX488-200-CSB $488 \mathrm{~nm}$ and Cobolt Flamenco $660 \mathrm{~nm}$ ). Each beam profile width was adjusted by an individual Keplarian telescope (not shown in the sketch of Figure 1g) and spatial overlay was achieved by a dichroic mirror (DMPL505; Thorlabs).

Conventional dSTORM imaging was performed on an inverted setup build around a microscope body (IX-71, Olympus) using a 60X NA 1.49 oil-immersion objective lens (Apo $\mathrm{N}, 60 \mathrm{X}$ NA 1.49; Olympus), described in detail elsewhere ${ }^{50}$. Briefly, laser light was emitted from an argon-krypton ion laser (Innova 70C, Coherent) and the $647 \mathrm{~nm}$ laser line was selected by an acousto-optic tunable filter (AOTFnC-VIS-TN 1001; AA Opto Electronic). Fluorescence emission was captured by an EMCCD camera (iXon DV887DCS-BV; Andor) with backprojected pixel widths and heights of $112 \mathrm{~nm}$ through two emission filters (LP02647RU-25; Semrock, ET700/75m; Chroma).

The TIRF excitation profile on the commercial OMX v4 Blaze system (GE Healthcare) was recorded using the ring TIRF mode and the field concentrator as the recommended illumination mode for dSTORM imaging. The microscope was equipped with a $642 \mathrm{~nm}$ 
excitation laser, an oil-immersion TIRF objective lens (60x NA1.49 ApoN, Olympus), and a sCMOS camera.

\section{Waveguide chip fabrication.}

The $\mathrm{Ta}_{2} \mathrm{O}_{5}$ waveguides were fabricated by sputtering a thin layer (150 to $220 \mathrm{~nm}$ ) of $\mathrm{Ta}_{2} \mathrm{O}_{5}$ onto a silicon dioxide $\left(\mathrm{SiO}_{2}\right)$ substrate. Magnetron sputtering was employed and the deposition was optimized using an iterative process. The parameters that were optimized for the waveguide parameters were substrate temperature, magnetron power and the oxygen flow rate. The optimized parameters chosen were $200^{\circ} \mathrm{C}$ substrate temperature, magnetron power of $300 \mathrm{~W}$ and $\mathrm{O}_{2}$ and Ar flow rates of 5 and $20 \mathrm{sccm}$, respectively. Post-deposition, standard photolithography was used to deposit photoresist for marking the waveguide geometry and ion-beam milling was used to fabricate the challenged waveguides (strip and rib). For rib waveguides, the slab was only partially etched down, while for strip the slab was etched completely down to the substrate. The sidewall roughness, which is the major source of propagation loss, was reduced by optimization of the ion-beam milling process. Finally, plasma etching was performed to remove the remaining photoresist from the photolithography step. Post-fabrication annealing for 5 hours at $600^{\circ} \mathrm{C}$ was employed to reduce the propagation losses.

The $\mathrm{Si}_{3} \mathrm{~N}_{4}$ waveguides were fabricated first by thermally growing a silica layer with a thickness of $2 \mu \mathrm{m}$ on silicon wafers. The thin $\mathrm{Si}_{3} \mathrm{~N}_{4}$ layer (150 nm thick) was deposited using low-pressure chemical vapor deposition (LPCVD) at $800^{\circ} \mathrm{C}$. Post-deposition, standard photolithography was used to deposit photoresist for marking the waveguide geometry, followed by reactive ion etching (RIE) to fabricate the challenged waveguides (strip and rib). The unwanted photoresist was then removed and a top cladding layer was deposited by plasma-enhanced chemical vapor deposition (PECVD) at $300^{\circ} \mathrm{C}$ of thickness $1.5 \mu \mathrm{m}$. At imaging areas, the top cladding was opened using RIE and wet etching to enable seeding of the cells. Further details of the fabrication process for $\mathrm{Ta}_{2} \mathrm{O}_{5}$ and $\mathrm{Si}_{3} \mathrm{~N}_{4}$ waveguides can be found elsewhere ${ }^{37}, 51$.

Before attaching cells onto the waveguides, the chip was thoroughly cleaned by submerging the chip in $5 \%(\mathrm{v} / \mathrm{v})$ Hellmanex (Sigma Aldrich) for 10 minutes at $70^{\circ} \mathrm{C}$. Hellmanex was removed using distilled $\mathrm{H}_{2} \mathrm{O}$, and another cleaning step using isopropanol (Sigma Aldrich) was performed before rinsing once more with $\mathrm{dH}_{2} \mathrm{O}$.

\section{Sample preparation.}

Sprague Dawley male rats (Scanbur) were kept under standard conditions and fed standard chow ad libitum (Scanbur). The experimental protocols were approved by the Norwegian National Animal Research Authority (NARA) in accordance with the Norwegian Animal 
Experimental and Scientific Purposes Act of 1986. The rats (body weight 150-300 g) were anesthetized with a mixture of medetomidin (Domitor vet, Orion) and ketamine (Ketalar, Pfizer) and LSECs were isolated and purified as described ${ }^{36}$. Cleaned waveguide chips were coated with human fibronectin $(50 \mu \mathrm{g} / \mathrm{ml})$ for $10 \mathrm{~min}$ at room temperature. Isolated LSECs in RPMI 1640 were seeded on the fibronectin coated waveguide chips and incubated for $1 \mathrm{~h}$ at $37^{\circ} \mathrm{C}$, followed by a washing step to remove non-attached cells and debris and allowed to spread their cytoplasm for another $2 \mathrm{~h}$. The cells were then washed with phosphate buffered saline (PBS) and fixed with 4\% paraformaldehyde (PFA) in PBS and $0.02 \mathrm{M}$ sucrose, $\mathrm{pH} 7.2$ for 30 minutes. Following fixation, the cell membranes were permeabilized with $0.1 \%$ Triton $X$ in PBS for 10 min at RT. Actin was stained with Atto 488-phalloidin (Sigma Aldrich), 1:400 dilution in PBS by 20 min incubation at RT. Membranes were stained with CellMask Deep Red (Life Technologies), 1:1000 dilution in PBS by 10 min incubation at RT. Tubulin was either stained with 1:400 alpha-tubulin antibody, Alexa Fluor 488 (ThermoFisher Scientific), at 1:400 dilution in PBS containing $5 \%$ bovine serum albumin, fraction $\vee$ (AppliChem) by 20 min incubation at RT or with mouse anti beta-tubulin (Sigma Aldrich), at 1:400 dilution in PBS/BSA for $1 \mathrm{~h}$ at RT, followed by goat anti mouse Alexa Fluor 647 (ThermoFisher Scientific) at the same concentration as the primary antibody, for $1 \mathrm{~h}$ at RT.

Dye surfaces were prepared by submerging approx. $50 \mathrm{nM}$ Alexa 647 in $0.05 \%$ Poly-Llysine (Sigma Aldrich) and incubating it for approx. $20 \mathrm{~min}$ on the waveguide chip or coverglass followed by an optional washing step. For measurements of the achievable localization precision using high and low NA objective lenses on the inverted conventional setup, $200 \mathrm{~nm}$ TetraSpeck Microspheres (Thermo Fisher Scientific) were diluted at approx. 1:50 in Vectashield H-1000 (Vector Laboratories).

Alexa 647-labelled R50 nanorulers (Gattaquant) were prepared on the waveguide by subsequently placing a Polydimethylsiloxane (PDMS) chamber on the surface, washing it thrice with PBS, incubating it with $0.5 \mathrm{mg} / \mathrm{ml}$ BSA-biotin (Sigma Aldrich) in PBS for $5 \mathrm{~min}$, incubating it with $0.5 \mathrm{mg} / \mathrm{ml}$ Neutravidin (VWR) in PBS for $5 \mathrm{~min}$, and incubating it with $0.5 \mu \mathrm{l}$ R50 stock solution in $10 \mu \mathrm{l} 60 \mathrm{mM}$ Magnesium chloride in PBS for $5 \mathrm{~min}$. Each step beginning from the BSA-biotin incubation was followed by two or three times washing with $60 \mathrm{mM}$ Magnesium chloride $\left(\mathrm{MgCl}_{2}\right)$ in PBS. For imaging on the conventional inverted setup, the sample was prepared on a coverglass (\#1.5) (Thermo Fisher Scientific Gerhard Menzel) by placing a PDMS chamber on the surface, washing it thrice with PBS, incubating it with 5 $\mathrm{mg} / \mathrm{ml} \mathrm{BSA-biotin} \mathrm{(Sigma} \mathrm{Aldrich)} \mathrm{in} \mathrm{PBS} \mathrm{for} 5 \mathrm{~min}$, washing it thrice with in PBS, incubating it with $0.5 \mathrm{mg} / \mathrm{ml}$ Neutravidin (Thermo Fisher Scientific) in PBS for $10 \mathrm{~min}$, and incubating it with $2.5 \mu \mathrm{l} \mathrm{R} 50$ stock solution in $25 \mu \mathrm{l} 10 \mathrm{mM}$ Magnesium chloride in PBS for $1 \mathrm{~h}$. Each step 
beginning from the Neutravidin incubation was followed by three times washing with $10 \mathrm{mM}$ Magnesium chloride in PBS.

Imaging buffer was prepared by mixing $\mathrm{H}_{2} \mathrm{O}$-based $22.5 \mu \mathrm{l}$ oxygen scavenger system solution (based on glucose oxidase (Sigma-Aldrich) and catalase (Sigma-Aldrich) ${ }^{52}$, with 30 $\mu$ PBS. The buffer was supplemented with $2 \mathrm{mM}$ cyclooctatetraene (COT) (Sigma Aldrich) in case of Alexa 647 imaging and with different concentrations of Mercaptoethylamine (MEA) (Sigma Aldrich). An MEA concentration of $95 \mathrm{mM}$ was used in case of CellMask Deep Red and Atto 488 imaging which we found to yield good blinking behavior on the waveguide for both CellMask Deep Red and Atto 488. In case of all Alexa 647 imaging experiments on the waveguide, we used an MEA concentration of $20 \mathrm{mM}$. The buffer for Alexa 647-labelled DNA nanorulers on the conventional inverted microscope was prepared from $\mathrm{D}_{2} \mathrm{O}$-based components, supplemented with $45 \mathrm{mM}$ Tris, and contained MEA at a concentration of 10 $\mathrm{mM}$. The same MEA concentration with additional $2 \mathrm{mM}$ COT was used in $\mathrm{H}_{2} \mathrm{O}$-based buffer to record the example raw dSTORM data on the conventional inverted setup. Sub-diffraction sized, fluorescent $100 \mathrm{~nm}$ TetraSpeck Microspheres (Life Technologies) at a low concentration (approx. 1:5000 from stock) aid algorithmic drift correction of super-resolved images.

To prevent light from coupling into the coverslip, we mounted a custom made PDMS chamber of $120 \mu \mathrm{m}$ height on top of the chip to lift the coverglass (\#1.5) (Thermo Fisher Scientific Gerhard Menzel) except for the experiment shown in Fig. 5 and Supplementary Fig. 17. It was verified that only light guided by the waveguide excites fluorescence (Supplementary Fig. 5, Video 1). The chamber additionally served as a vessel for the imaging buffer. Alternatively, the buffer was held by capillary action between the chip and the coverslip directly deposited on the waveguide. In the latter case, a small piece of black PDMS of significantly greater height (approx. $2 \mathrm{~mm}$ ) was applied close to the input facet to block any unguided light before the coverslip (experimental results shown in Fig. 5 and Supplementary Fig. 17). This PDMS was manufactured such that a thin layer touching the waveguide was transparent, to prevent light absorption by the black PDMS.

\section{Image acquisition and data analysis.}

Several hundred frames were acquired at relatively low power at the input facet for waveguide-based imaging. During acquisition, the coupling objective lens was oscillated along the input facet. These image stacks were used as input for the ESI reconstruction. Alternatively, averaging over the acquired frames yields a diffraction-limited image of the sample. Exposure times for dSTORM ranged from 10 to $160.84 \mathrm{~ms}$. For multi-colour 
dSTORM images (Fig. 5, Supplementary Fig. 17), different channels were recorded subsequently. A detailed register of imaging conditions is found in Supplementary Table 1. Image processing and analysis was carried out using $\mathrm{Fiji}^{33}$ and Matlab (Mathworks). ESI reconstruction and postprocessing was performed using the Fiji plugin $\mathrm{ESI}^{7}$. Reconstructions were run in two iterations at second ESI order with an intermediate gamma correction step using a gamma factor of 0.5 to compensate for non-linear intensity amplification. For dSTORM reconstruction and postprocessing the Fiji plugin ThunderSTORM ${ }^{54}$ was used. Localizations were filtered for unphysical values of fitted FWHM width and localization precision. Non-linear drift correction was performed via cross-correlation of substacks. Rendered dSTORM images were convolved with a 2D Gaussian kernel (see Supplementary Table 1 for kernel width) in some cases. The look up tables and intensity values for shown figures were adjusted to achieve appropriate contrast including gamma correction, however all quantifications were made on linearly scaled images. For localization precision calculations ${ }^{32}$, we used custom written Matlab code. FRC analysis was carried out using the Fiji plugin $\mathrm{FRC}^{33}$. Diffraction limited images were computed by averaging over all acquired frames recorded prior to dSTORM imaging or the raw data also used as input for the ESI reconstruction.

Evanescent field intensities on the waveguide were measured using the fluorescent response of single sub-diffraction sized $100 \mu \mathrm{m}$ TetraSpeck Microspheres (Life Technologies) as intensity sensors. A reference measurement was conducted by imaging the microspheres on the conventional inverted setup in epi-illumination mode. The photon response as a function of the illumination intensity was recorded and corrected for multiple factors including laser power, camera quantum efficiency, single-frame exposure time, the Gaussian-like beam profile in epi-illumination, and the waveguide breadth in waveguide illumination. Similar measurements were carried out on the waveguide to find the photon response as a function of the power at the input facet of the waveguide and estimate the corresponding evanescent field intensity. Though several factors have been corrected for, these numbers should only be taken as a rough estimation as the efficiency of coupling light into the waveguide differs between different chips.

\section{ESI simulation.}

Chip-based ESI was simulated by generating raw data of 200 frames in Matlab (Mathworks) as input for the ESI plugin. Multi-mode interference was modelled as sinusoidal patterns rendered to a fine grid with equally distributed random phases and equally distributed pattern wavelengths between $280 \mathrm{~nm}$ and $840 \mathrm{~nm}$. The lower bound wavelength of $280 \mathrm{~nm}$ corresponds to a FWHM of $140 \mathrm{~nm}$ in the multi-mode interference pattern that we have measured for excitation with the laser of $660 \mathrm{~nm}$ vacuum wavelength (Supplementary Fig. 
11). The fluorescent response of emitter pairs at different distances was modelled by assuming a linear response to the illumination, i.e. taking the respective illumination intensity at the emitters' positions as the fluorescent response in each frame. This signal was convolved with a Gaussian point-spread-function with a FWHM of $\lambda / 2 N A$ with the emission wavelength $\lambda=678 \mathrm{~nm}$ and the numerical aperture $\mathrm{NA}=1.2$, and afterwards scaled down to a grid with pixel widths and heights of $75.9 \mathrm{~nm}$. Shot noise was modelled as Poisson noise and the signal was converted from counts to photons at 2.4 photons per count at a maximum of 500 photons per pixel. To consider additional camera noise, we used the sCMOS camera to record 200 frames with no illumination on the detector and added these to the modelled frames. The resulting image stack was imported to Fiji for ESI analysis.

\section{Data availability statement}

The data that support the images and plots within this paper and other findings of this study are available from the corresponding author upon reasonable request. 


\section{REFERENCES FOR METHODS}

7 Yahiatene, I., Hennig, S., Muller, M. \& Huser, T. Entropy-Based Super-Resolution Imaging (ESI): From Disorder to Fine Detail. Acs Photonics 2, 1049-1056, doi:10.1021/acsphotonics.5b00307 (2015).

32 Endesfelder, U., Malkusch, S., Fricke, F. \& Heilemann, M. A simple method to estimate the average localization precision of a single-molecule localization microscopy experiment. Histochemistry and cell biology 141, 629-638 (2014).

33 Nieuwenhuizen, R. P. et al. Measuring image resolution in optical nanoscopy. Nat Methods 10, 557-562, doi:10.1038/nmeth.2448 (2013).

36 Smedsrød, B. \& Pertoft, H. Preparation of pure hepatocytes and reticuloendothelial cells in high yield from a single rat liver by means of Percoll centrifugation and selective adherence. Journal of leukocyte biology 38, 213-230 (1985).

37 Ahluwalia, B. S. et al. Fabrication of submicrometer high refractive index Tantalum Pentoxide waveguides for optical propulsion of microparticles. Photonics Technology Letters, IEEE 21, 1408-1410 (2009).

50 Diekmann, R. et al. Nanoscopy of bacterial cells immobilized by holographic optical tweezers. Nat Commun 7, 13711, doi:10.1038/ncomms13711 (2016).

51 Prieto, F. et al. An integrated optical interferometric nanodevice based on silicon technology for biosensor applications. Nanotechnology 14, 907-912, doi:10.1088/0957-4484/14/8/312 (2003).

52 van de Linde, $S$. et al. Direct stochastic optical reconstruction microscopy with standard fluorescent probes. Nature protocols 6, 991-1009 (2011).

53 Schindelin, J. et al. Fiji: an open-source platform for biological-image analysis. Nature methods 9, 676-682 (2012).

54 Ovesny, M., Krizek, P., Borkovec, J., Svindrych, Z. \& Hagen, G. M. ThunderSTORM: a comprehensive ImageJ plug-in for PALM and STORM data analysis and superresolution imaging. Bioinformatics 30, 2389-2390, doi:10.1093/bioinformatics/btu202 (2014). 


\section{Chip-based wide field-of-view nanoscopy}

Robin Diekmann ${ }^{\# 1}$, Øystein I. Helle ${ }^{\# 2}$, Cristina I. $\varnothing_{i e^{2}}$, Peter McCourt ${ }^{3}$, Thomas R. Huser ${ }^{1,4}$, Mark Schüttpelz ${ }^{1}$ and Balpreet S. Ahluwalia ${ }^{2}$

\# These authors contributed equally to this work.

1 Department of Physics, Bielefeld University, 33615 Bielefeld, Germany.

2 Department of Physics and Technology, UiT-The Arctic University of Norway, 9037 Tromsø, Norway.

3 Department of Medical Biology, UiT-The Arctic University of Norway, 9037 Tromsø, Norway.

4 Department of Internal Medicine and NSF Center for Biophotonics, University of California, Davis, Sacramento, CA 95817, USA

Correspondence should be addressed to M.S. (schuettp@physik.uni-bielefeld.de) or B.S.A. (balpreet.singh.ahluwalia@uit.no). 
Supplementary Figure 1 | The resolution of chip-based dSTORM acquired with a 60x NA 1.2 objective lens is investigated using different methods. The lateral profile along a microtubule (Fig. 2a,b) shows two distinct peaks corresponding to the separation distance of the shell-like structure of an antibody stained microtubule filament. Their distance of $(47 \pm 5) \mathrm{nm}$ is measured by fitting the sum of two Gaussians (a). Their FWHM values of $(49 \pm 7) \mathrm{nm}$ and $(40 \pm 5) \mathrm{nm}$ affirm the resolution below $50 \mathrm{~nm}$. (b) Consistently, we find a Fourier-ring-correlation resolution ${ }^{33,34}$ of $(41.3 \pm 2.3) \mathrm{nm}$ for the dSTORM image of Fig. 2a. Localization precision values serve as lower bounds for the obtainable resolution. The localization precision distribution found from the photon statistics ${ }^{55,56}$ using the reconstruction software $^{54}$ shows a mean value of $8.5 \mathrm{~nm}$ (a), which is in good agreement with the alternative method of using the statistics of nearest neighbours in adjacent frames ${ }^{32}$ that shows a mean localization precision of $(8.3 \pm 0.2) \mathrm{nm}$. Note that the localizations were filtered for localization precisions less than $13 \mathrm{~nm}$.

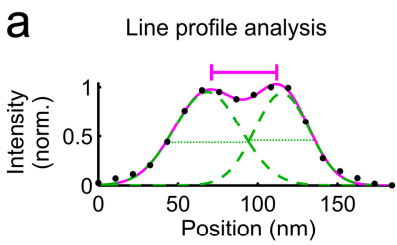

Peak-to-peak distance: $47 \mathrm{~nm}$ $\mathrm{FWHM}_{1}: 49 \mathrm{~nm}$ $\mathrm{FWHM}_{2}: 40 \mathrm{~nm}$

b

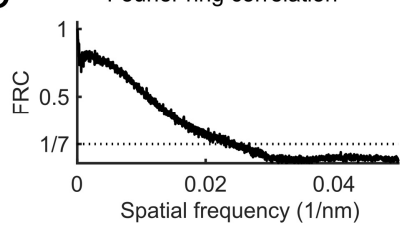

FRC resolution:
C

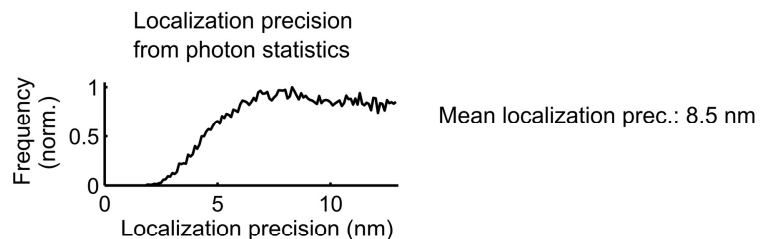
$(41.3 \pm 2.3) \mathrm{nm}$

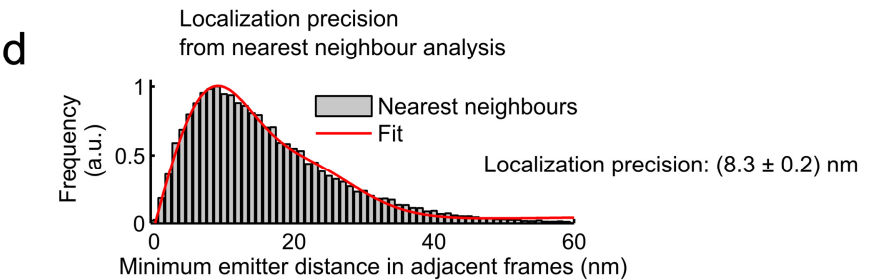


Supplementary Figure 2 | Mechanical drift in the setup for chip-based dSTORM. Subdividing the localizations table into multiple stacks, calculating their cross-correlation, and interpolating between the discrete points allows for finding the drift that occurred during the acquisition of the raw data ${ }^{54,57}$. The graph shows the mechanical drift during the chipbased measurement shown in Fig. 2c, which corresponds to about $500 \mathrm{~nm}$ after about 25,000 frames. Although this value lies significantly above the obtained resolution it can easily be corrected for in the localizations table before the image is rendered.

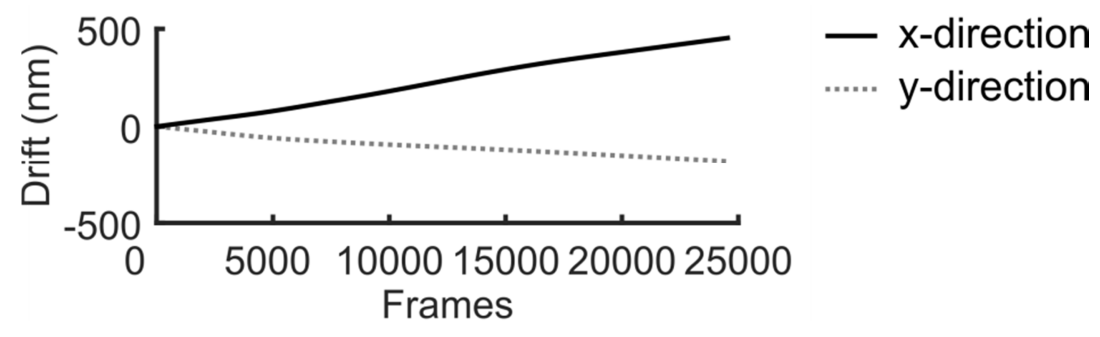


Supplementary Figure 3 | Examples of single frames from dSTORM imaging of Alexa 647 on a conventional upright setup with objective-based TIRF excitation (a) and waveguide chip-based dSTORM (b) show comparable quality of the raw data. (Scale bars, $1 \mu \mathrm{m}$.)

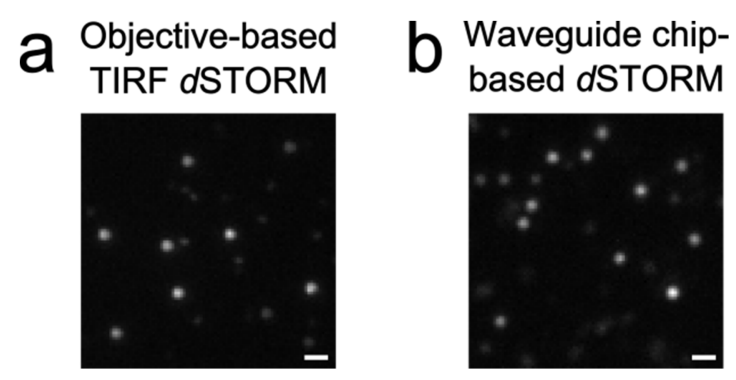


Supplementary Figure 4 | The waveguide allows for TIRF-like imaging, providing optical sectioning via evanescent excitation. We measured the penetration depth of the evanescent field of the waveguide to be $(190 \pm 59) \mathrm{nm}$ when using illumination of $660 \mathrm{~nm}$ vacuum wavelength, and (172 \pm 54$) \mathrm{nm}$ when using illumination of $488 \mathrm{~nm}$ vacuum wavelength.

Measurements were conducted by using beads with a mean diameter of $8.18 \mu \mathrm{m}$, whose surface was labelled with Atto $647 \mathrm{~N}$ or Atto 488, respectively. Measuring the individual bead diameter and finding its centre allows for calculating the height of its surface over the waveguide. The spatially dependent strength of the evanescent field is then measured by the fluorescence intensity ${ }^{58,59}$. The plot shows a typical result, which can be used to determine the penetration depth by fitting an exponentially decaying function to the data points of a height over the waveguide ranging from 0 to $300 \mathrm{~nm}$.

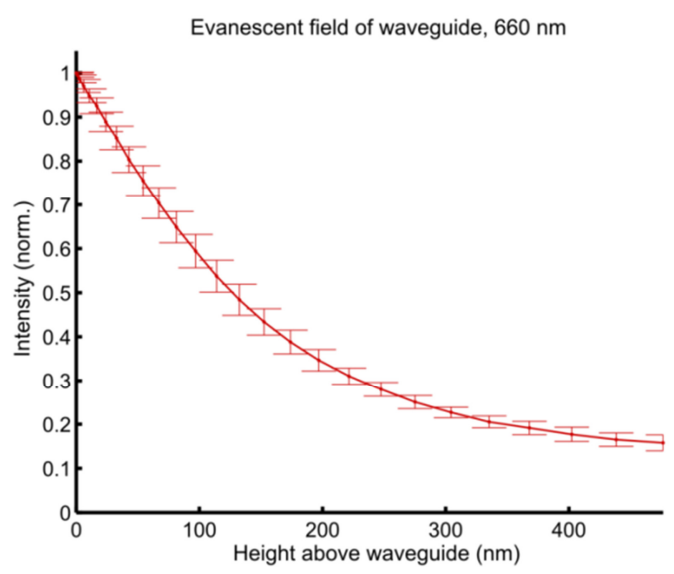


Supplementary Figure $\mathbf{5}$ | Fluorescence is excited only by the evanescent field of the waveguide and not by scattered light. We verified this by laterally shifting the strip waveguide chip, which leads to a loss in coupling accompanied with a loss of most of the fluorescence signal from fluorescent beads (200 nm diameter). This is recovered by moving the waveguide back to its original location (Video 1).

This figure shows the first frame of the video. As a guide to the eye, the approximate position of the edges of the waveguide (grey line) and the coupling objective lens are shown.

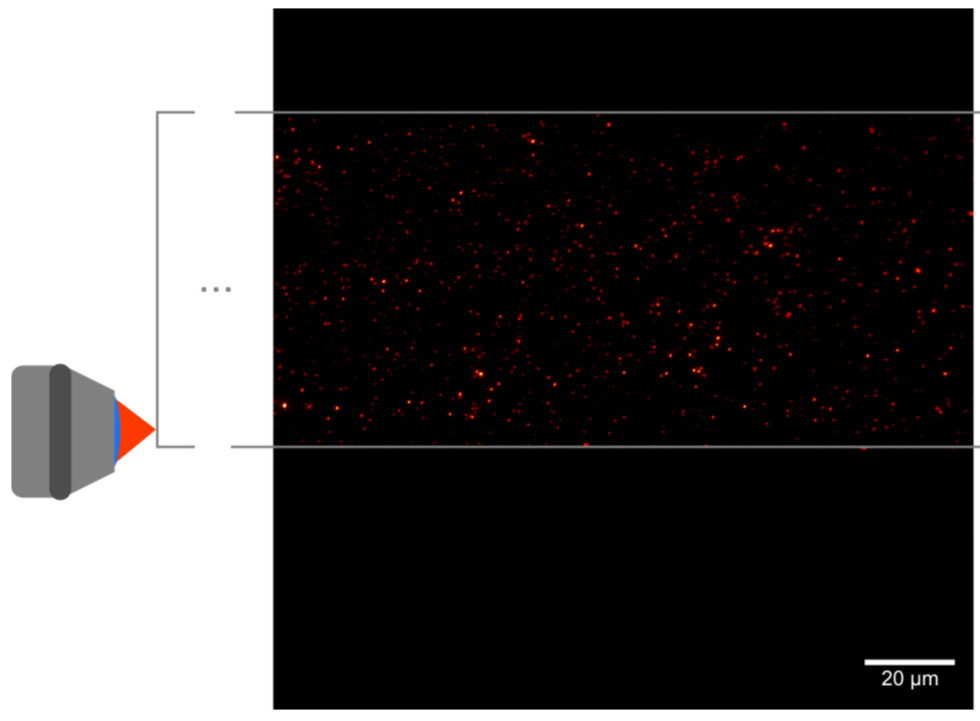


Supplementary Figure 6 | Resolution estimates for chip-based dSTORM using a 20x NA 0.45 objective lens. (a) The separation distance between adjacent tubulin filaments as shown in Fig. 2f,g. Fitting the sum of two Gaussians finds the distance of $138 \mathrm{~nm}$ for the distinct peaks. The resolution below $140 \mathrm{~nm}$ is confirmed by the FWHM values of $(99 \pm 14) \mathrm{nm}$ and $(105 \pm 21) \mathrm{nm}$ as well as a Fourier ring correlation ${ }^{33,34}$ resolution of $(122 \pm 10) \mathrm{nm}$ (b). This resolution is further verified by the mean localization precision estimates from both the photon statistics ${ }^{55,56}$ using the reconstruction software ${ }^{54}$ (c) and from nearest neighbour analysis ${ }^{32}$ (d). The nearest neighbour analysis was conducted on a ROI of $90 \mu \mathrm{m} \times 72 \mu \mathrm{m}$ size including the line profile of (a). Note that the localizations were filtered for localization precisions less than $60 \mathrm{~nm}$.

a

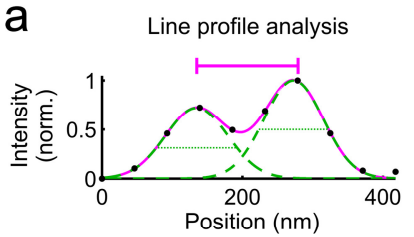

Peak-to-peak distance: $138 \mathrm{~nm}$ $\mathrm{FWHM}_{1}: 105 \mathrm{~nm}$ $\mathrm{FWHM}_{2}: 99 \mathrm{~nm}$

b

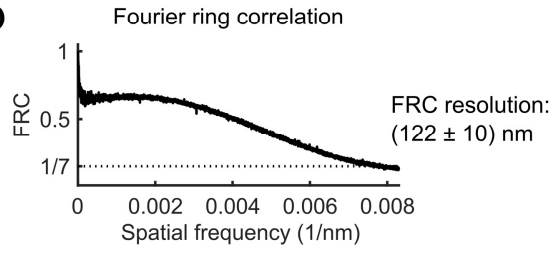

C

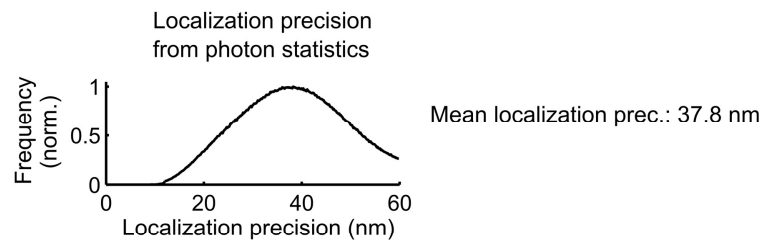

d Localization precision from nearest neighbour analysis

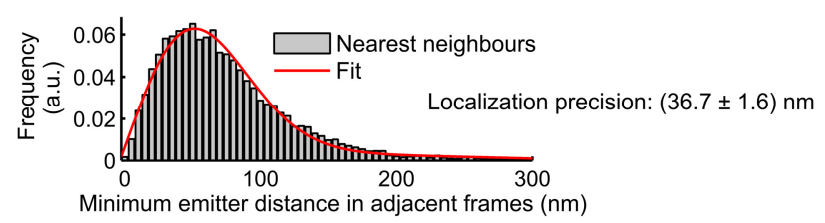


Supplementary Figure 7 | Different wavelengths can be combined simultaneously in chipbased dSTORM by overlaying them before coupling into the waveguide. This allows for effective photoswitching, here demonstrated for CellMask Deep Red. At frame 7650, the $488 \mathrm{~nm}$ laser is switched on, providing power at the input facet of the waveguide in addition to the $660 \mathrm{~nm}$ laser. Activation of the $488 \mathrm{~nm}$ laser line helps in switching CellMask Deep Red back to the fluorescent state, indicated by an increase by $33 \%$ in the number of mean localizations per 30 frames.

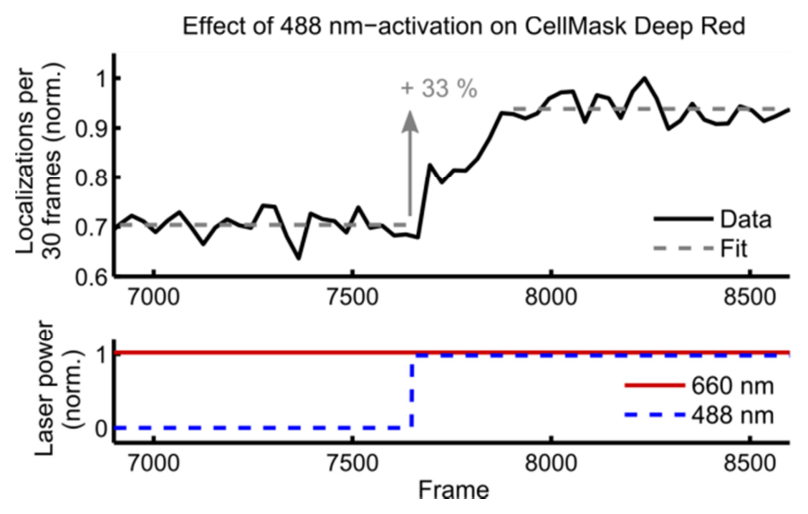


Supplementary Figure 8 | Diffraction limited illumination pattern measurements. (a) Dense dye labelling of the waveguide surface and averaging over 200 frames allows measuring the homogeneity of the illumination (b). Ignoring the edges, we find a modulation depth of $16 \%$ over the stretch of the waveguide (c) that is used for fluorescence excitation and singlemolecule photoswitching. A low modulation depth does not significantly influence the quality of the dSTORM image as discussed in ${ }^{35}$ and verified by our results as the structure of the illumination pattern is not visible in the reconstructed $d S T O R M$ images (Supplementary Fig. 9). (d,e) To compare the performance to a commercial setup for dSTORM imaging, we used ring-TIRF illumination on an OMX v4 Blaze system (GE Healthcare) equipped with a field concentrator. To limit the modulation depth of the illumination intensity to $16 \%$ in this case restricts the field of view to about $18 \mu \mathrm{m} \times 18 \mu \mathrm{m}(\mathrm{f})$. Outside this region, the illumination intensity drops significantly which limits the applicable FOV for dSTORM imaging. The illumination profile of waveguide TIRF excitation is less smooth, but its modulation does not become apparent in the dSTORM images. However, in this case the illuminated area is only limited by the waveguide design and no significant drop in illumination intensity is observed over the stretch of the waveguide in both lateral directions. (Scale bars, $10 \mu \mathrm{m}$.)
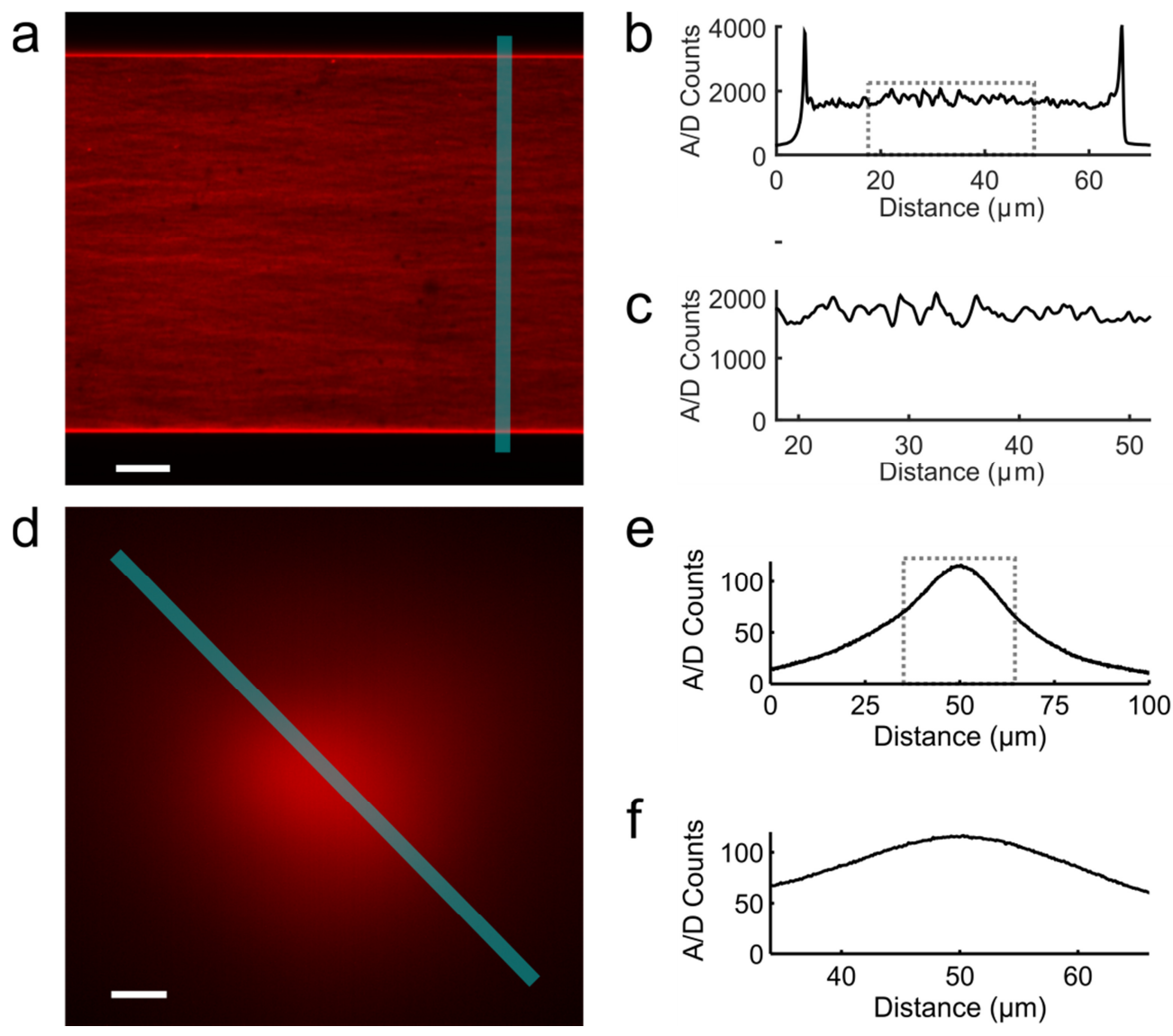
Supplementary Figure 9 | The use of broad waveguides (25 to $500 \mu \mathrm{m}$ ) results in multimode patterns that cause a laterally inhomogeneous evanescent field. Consequently, fluorescence excitation is non-uniform and the shape of the multimode pattern masks the imaged structures $(a, b)$. Oscillating the coupling objective in ranges that still maintain coupling leads to a movement of the multimode pattern (Video 2), (a) and (b) show a ROI from different frames of the video, allowing for correcting for the non-uniform excitation by averaging over multiple frames (c). In this case, we have averaged over 61 frames. During dSTORM imaging, the coupling objective lens is also oscillated. Accordingly, the pattern is not visible in the super-resolved reconstruction of the LSEC membrane (d). (Scale bar, $2 \mu \mathrm{m}$.

a

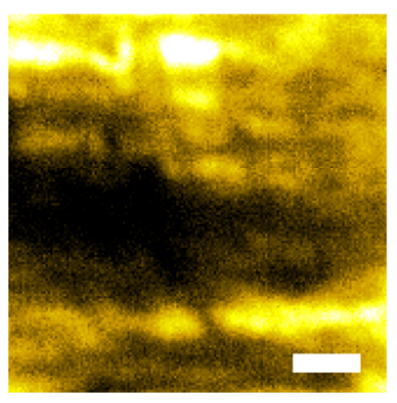

b

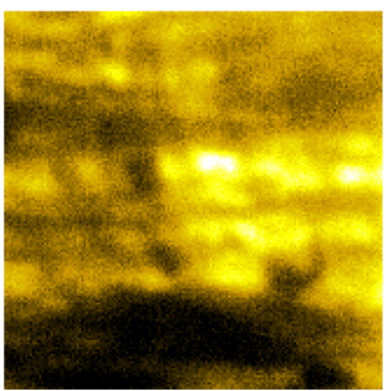

C

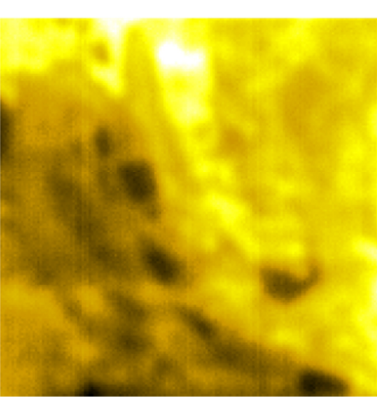

d

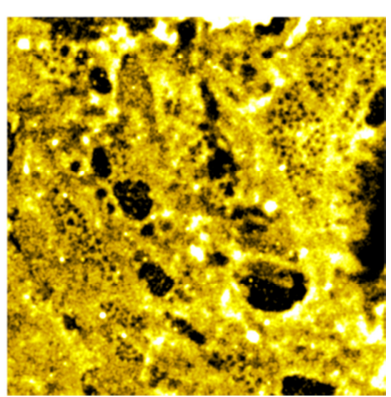


Supplementary Figure 10 | Simulation of chip-based ESI. We modelled a random phase sinusoidal illumination pattern with random fringe widths comparable to the measured values exciting emitter pairs at different distances (Methods). (a) The average signal over 200 frames shows that two emitters at the diffraction limit of $283 \mathrm{~nm}$ distance are just resolved (d), while emitters at $150 \mathrm{~nm}$ (c) and $125 \mathrm{~nm}$ (b) are not resolved. The ESI reconstruction results in a separation of the emitters at $150 \mathrm{~nm}$ distance (c), while the emitters at $125 \mathrm{~nm}$ distance are not separated (b), but the emitters at $283 \mathrm{~nm}$ distance become more clearly separated (d). (Scale bars, $1 \mu \mathrm{m}$.)

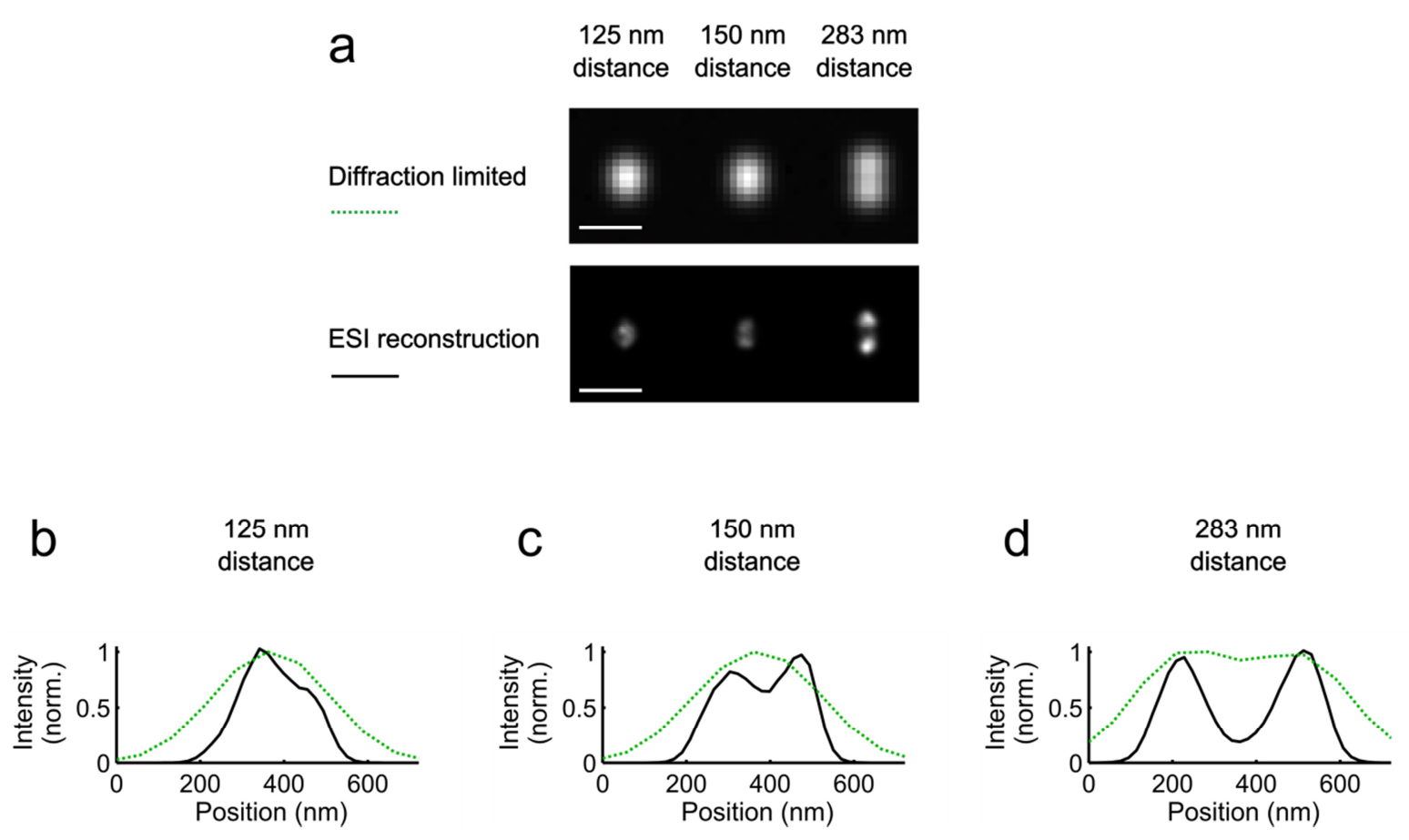


Supplementary Figure 11 | The multi-mode interference pattern of a strip waveguide imaged using dSTORM. A surface of Alexa 647 dye molecules was prepared on top of the waveguide and a dSTORM image was acquired giving the instant lateral field-distribution of the evanescent field. Interference fringes show structures down to $140 \mathrm{~nm}$ as seen in the line profiles. (Scale bar, $5 \mu \mathrm{m}$.)

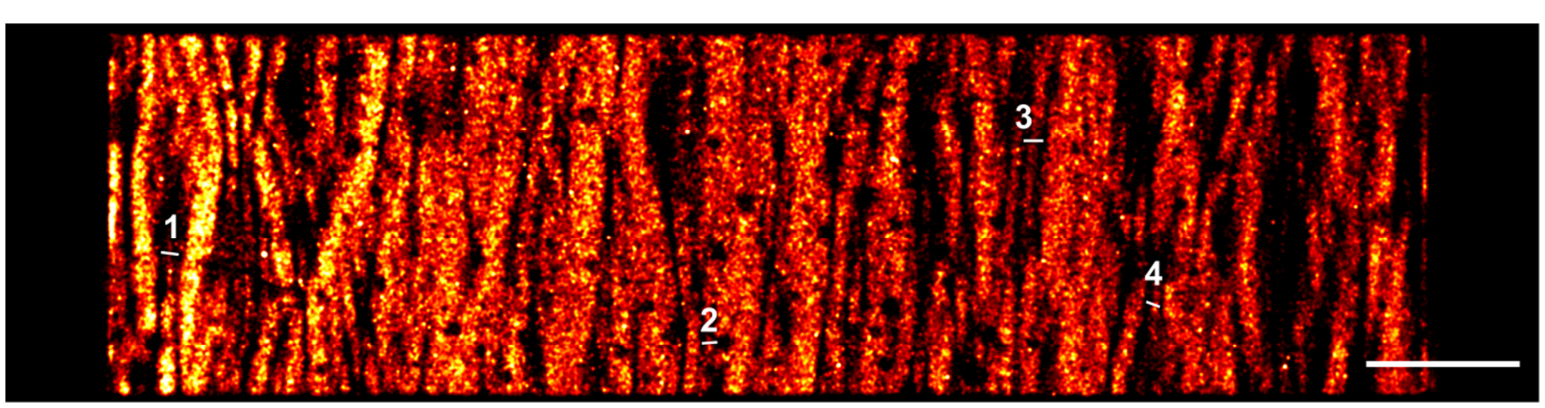

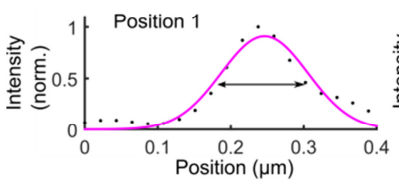

FWHM: $140 \mathrm{~nm}$

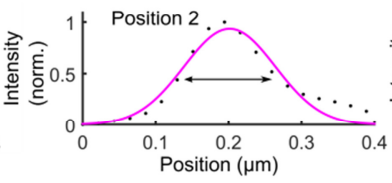

FWHM: $148 \mathrm{~nm}$

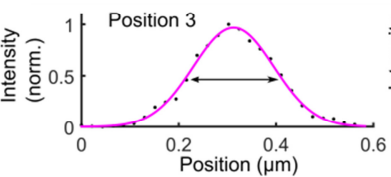

FWHM: $195 \mathrm{~nm}$

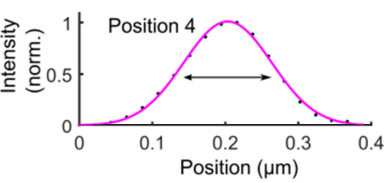

FWHM: $142 \mathrm{~nm}$ 
Supplementary Figure 12 | (a) Chip-based ESI reconstruction of tubulin in the LSEC shown in Fig 3b. (Scale bar, $5 \mu \mathrm{m}$.) (b) and (c) show the region marked by a white box in (a) for diffraction limited imaging and chip-based ESI, respectively. (Scale bars, $1 \mu \mathrm{m}$.) (d) Fitting a Gaussian function to the line plots for a tubulin filament shows a FWHM of $231 \mathrm{~nm}$ for the diffraction limited image and a FWHM of $104 \mathrm{~nm}$ for the ESI image.
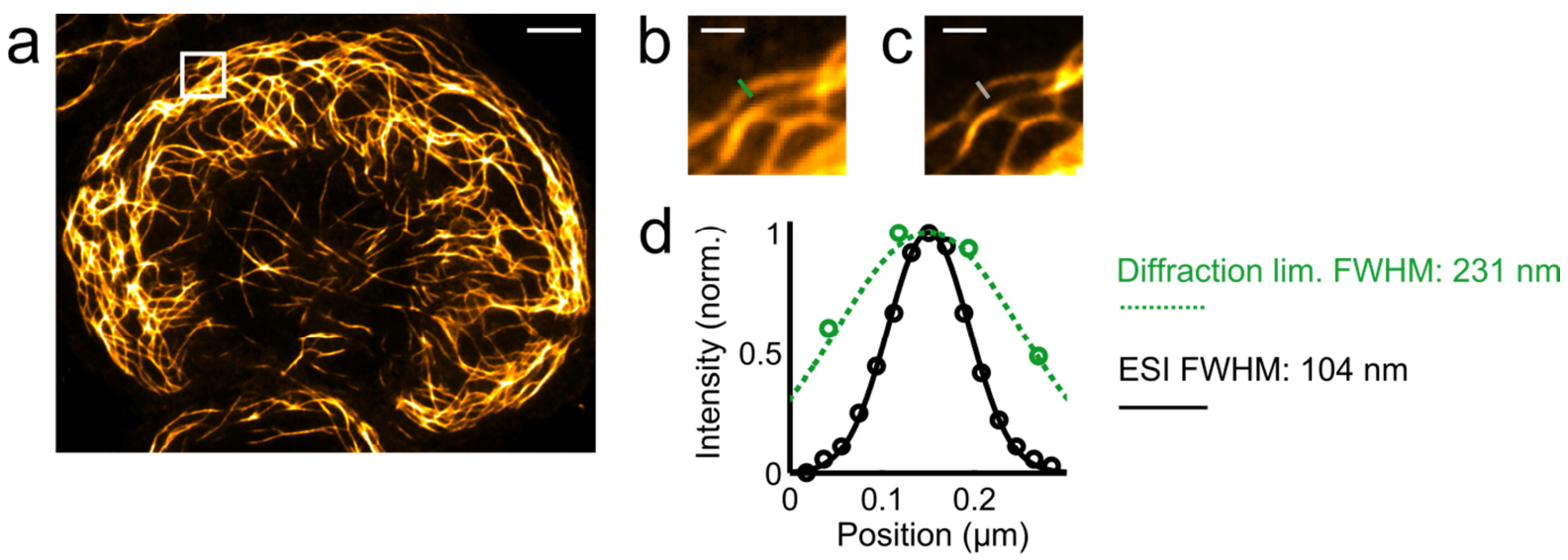
Supplementary Figure 13 | (a) Chip-based ESI reconstruction of actin in LSECs as shown in Fig 4b. (Scale bar, $20 \mu \mathrm{m}$.) (b) and (c) show the region marked with a white box in (a) for diffraction limited imaging and chip-based ESI, respectively. (Scale bars, $2 \mu \mathrm{m}$.) (d) Fitting a Gaussian function to the line plots for a single actin filament shows a FWHM of $691 \mathrm{~nm}$ for the diffraction limited image and a FWHM of $333 \mathrm{~nm}$ for the ESI image.
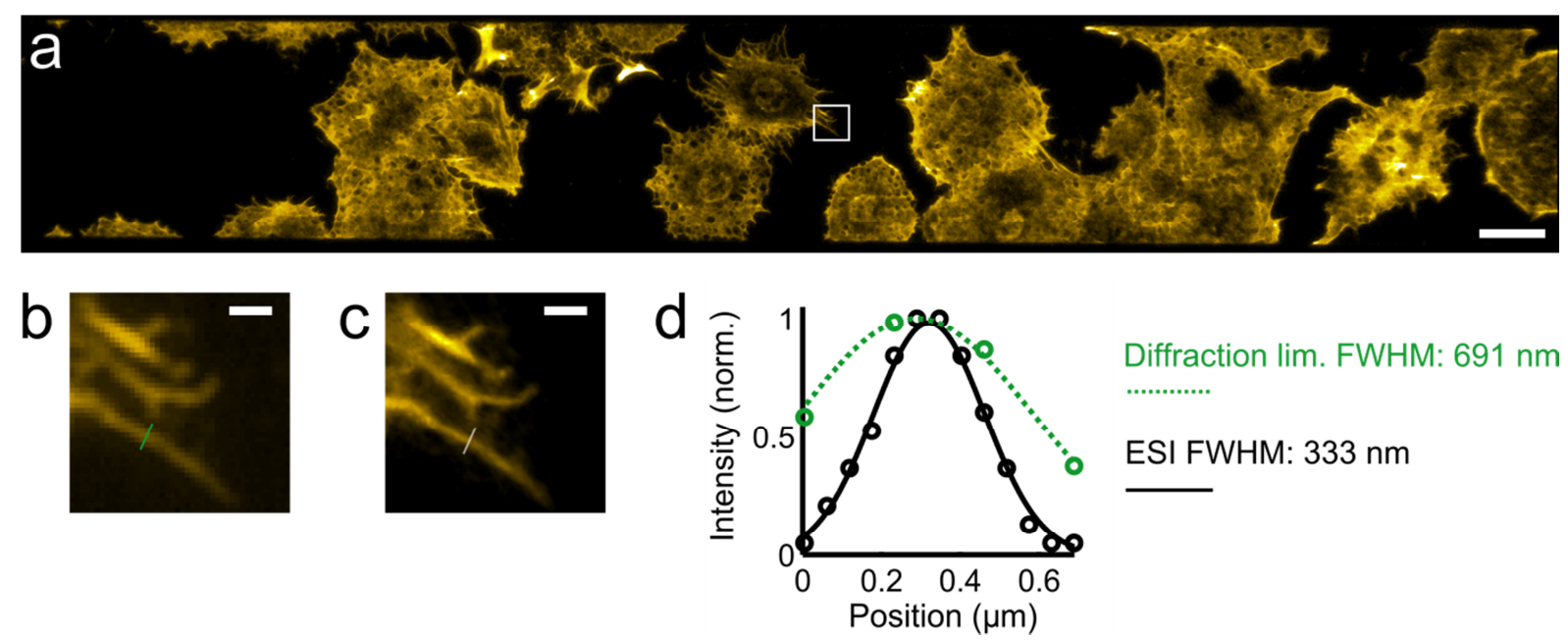

Diffraction lim. FWHM: $691 \mathrm{~nm}$

ESI FWHM: $333 \mathrm{~nm}$ 
Supplementary Figure 14 | Comparison of the experimentally achieved localization precision and the theoretical predictions for Atto 488 on the waveguide (a) and for fluorescent beads on a conventional inverted microscope (b). (c)-(f) show example raw data taken under different conditions and (g)-(j) show the corresponding reconstructed images for the entire stack of raw data. See Supplementary Note 2 for a discussion of the data. Within this figure, the following references are used: [a] = Thompson et al. $2002^{55}$, [b] = Mortensen et al. $2010^{56}$, [c] = Ovesny et al. $2014^{54}$, [d] = Endesfelder et al. $2014^{32}$. (Scale bars, $1 \mu \mathrm{m}$.)

a

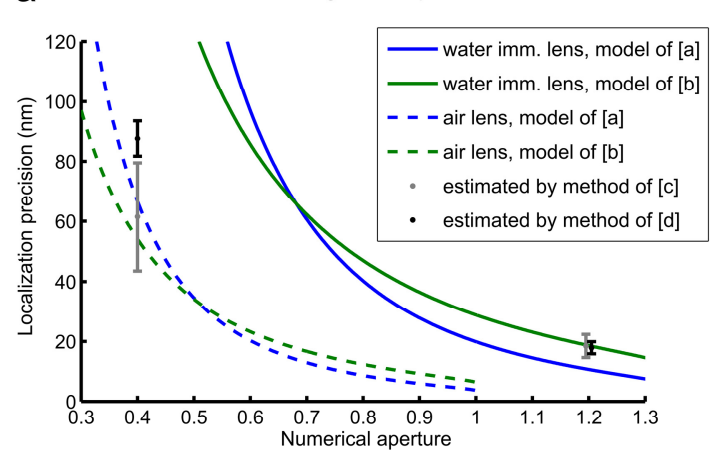

NA 0.4
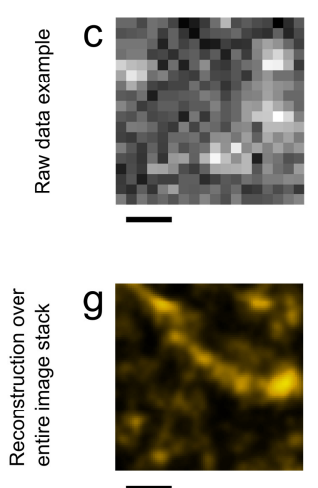

NA 1.2

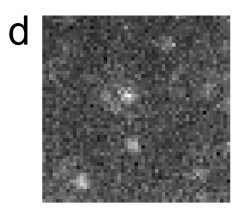

$\mathrm{h}$

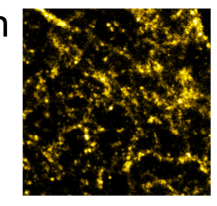

b

Conventional inverted microscope

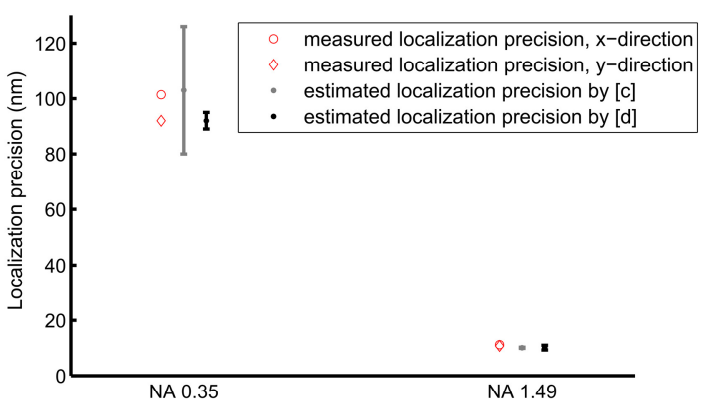

NA 0.35

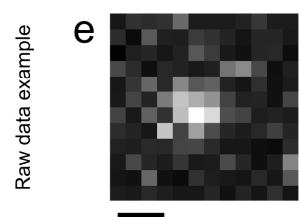

NA 1.49
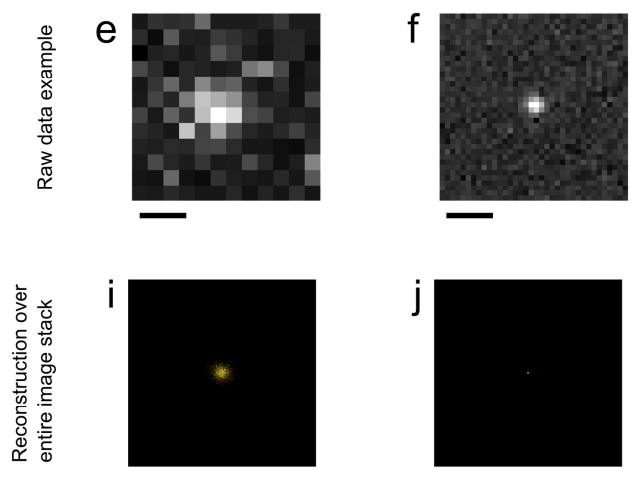
Supplementary Figure 15 | The comparison of the ROI shown in Fig. 4 f reconstructed from 3,000 frames and 10,044 frames, respectively, shows that 3,000 frames were sufficient to reconstruct the image with comparable quality using low magnification dSTORM (Supplementary Note 4). E.g. the two separated actin filaments at the edge of the cell are visible. Using a single frame exposure time of $160.84 \mathrm{~ms}$ and 3,000 frames leads to an overall acquisition time of 8 minutes, i.e. this approach enables a fast overview of a field of view of large width (Figure 4c) at a mean localization precision of $87.6 \mathrm{~nm}$ (Supplementary Figure 16). The short acquisition time helps to preserve fluorophores for subsequent reimaging of cells of interest using a higher NA/ higher magnification objective lens. However, we have found that using $95 \mathrm{mM}$ MEA in glucose oxidase and catalase based oxygen scavenger system buffer allows for at least 60 minutes dSTORM imaging on the waveguide for both Atto 488 and CellMask Deep Red. (Scale bars, $1 \mu \mathrm{m}$.)
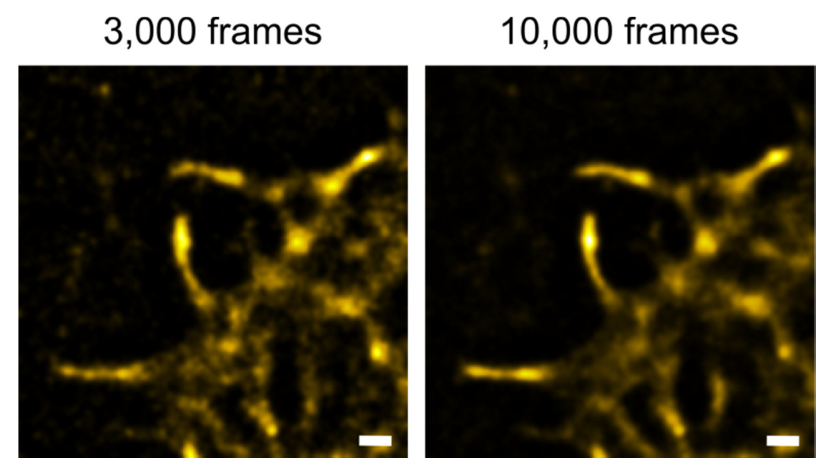
Supplementary Figure 16 | The use of a 20x NA 0.4 objective lens allows for imaging a field-of-view of approx. $0.46 \mathrm{~mm}$ width (Fig. 4c). The measured distance between actin filaments shows a resolution of at least $334 \mathrm{~nm}$ in the case of the low NA objective lens used for chip-based dSTORM. This result is confirmed by localization precision calculations ${ }^{32}$, estimating the average localization precision to be $87.6 \mathrm{~nm}$ as shown here.

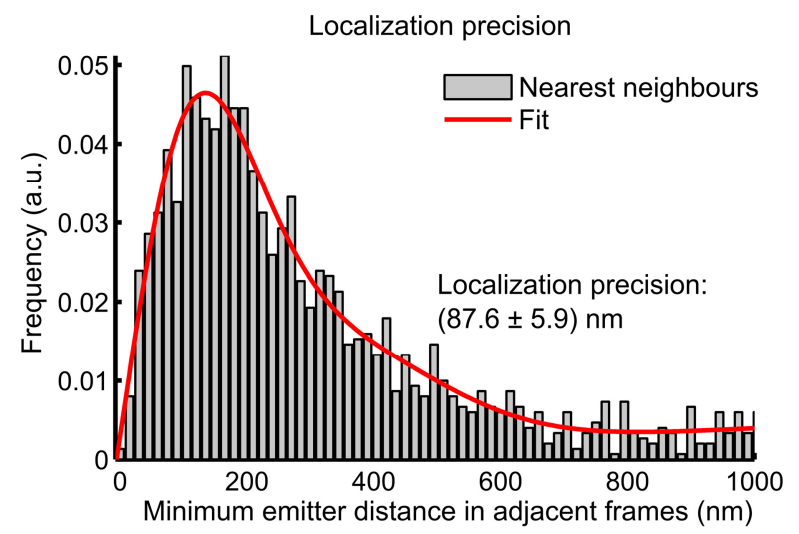


Supplementary Figure 17 | Comparison of diffraction limited images and dSTORM images of the cells shown in Fig. 5a. Diffraction limited images were recorded prior to the respective dSTORM measurement. (Scale bars, $10 \mu \mathrm{m}$.)

a Actin, Atto488-phallodin Widefield

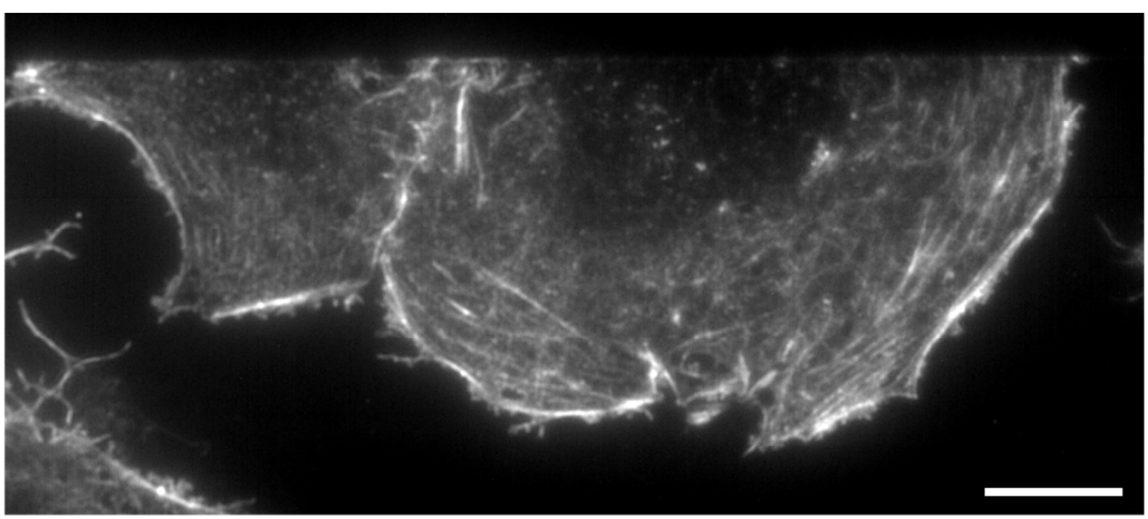

b Membrane, CellMask Deep Red Widefield

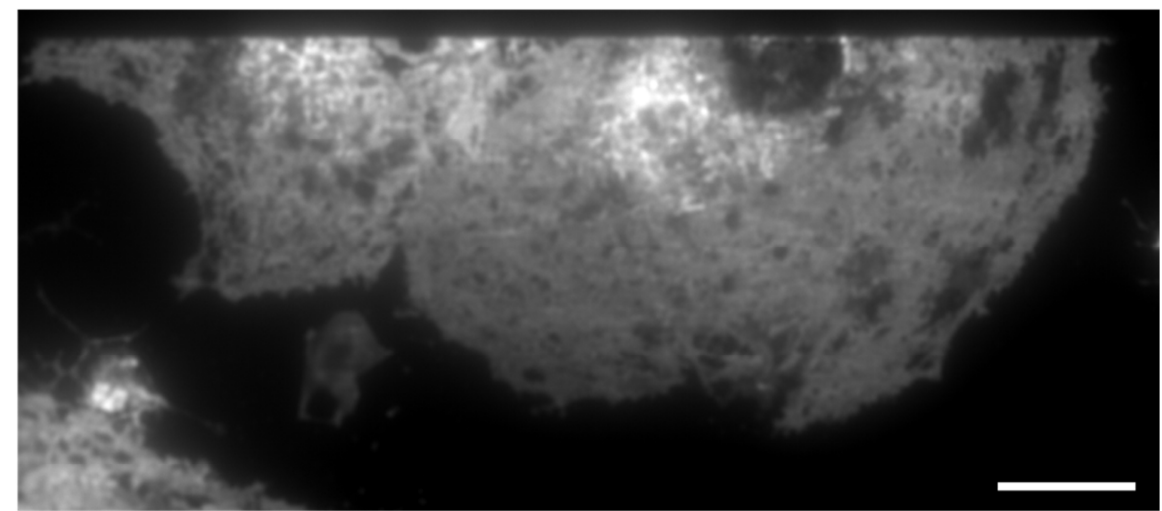

C Actin, Atto488-phallodin dSTORM

Membrane,

CellMask Deep Red dSTORM

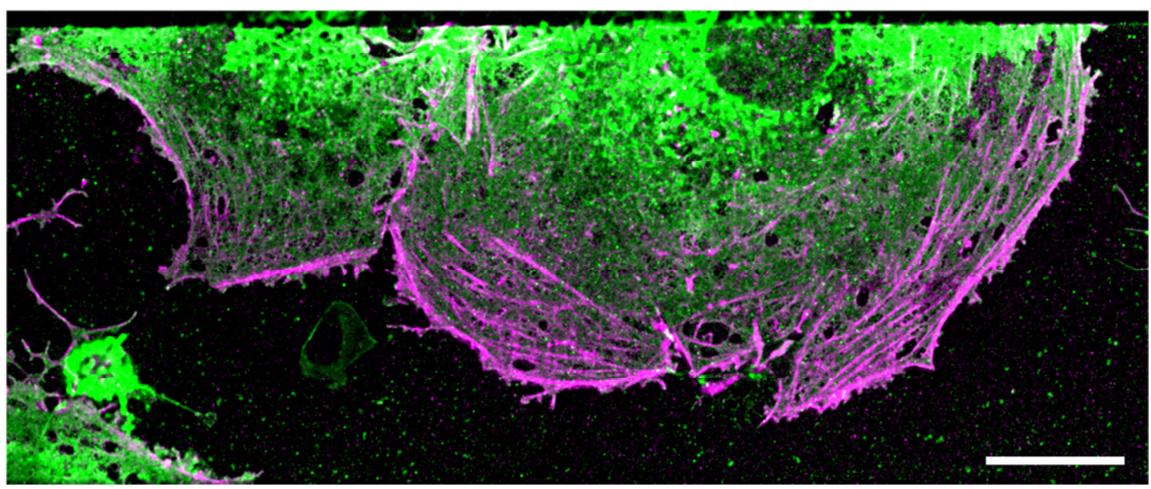




\section{Supplementary Note 1: Obtainable resolution for waveguide-based ESI}

In former work on fluctuating random pattern illumination by Kim et al. $^{39}$, the authors point out that achievable resolution enhancement with this technique depends on the size of the structures in the speckle pattern. In their work, diffraction limited illumination at a laser vacuum wavelength of $532 \mathrm{~nm}$ through a high NA 1.4 objective lens was used, resulting in speckle sizes in the illumination pattern down to $\lambda / 2 N A=190 \mathrm{~nm}$. The authors find a resolution of $144 \mathrm{~nm}$ when using SOFI with speckle pattern illumination, which gives a ratio between the theoretically smallest structures in the illumination pattern and the obtained resolution of 1.32. A similar value can be found for their results when using a low NA 0.4 objective lens. In this case, the speckle sizes go theoretically down to $665 \mathrm{~nm}$, while a resolution of $500 \mathrm{~nm}$ was demonstrated, resulting in a ratio of 1.33.

In our case, the interference pattern is generated using a laser of $488 \mathrm{~nm}$ vacuum wavelength and a waveguide with a refractive index of about 1.7. This results in a lower bound for the interference fringe period in the illumination pattern of about $\lambda / 2 n_{\text {eff }}=143 \mathrm{~nm}$. With this, a spatial resolution on the order of $110 \mathrm{~nm}$ (Fig. 3d, Supplementary Fig. 12) was achieved using a high NA 1.2 objective lens, which gives a ratio between the theoretically smallest illumination pattern sizes and the obtained resolution of 1.3 , and matches the previously reported results in this field. However, we found a lower ratio of 0.43 when using a low NA 0.4 objective lens, though the illumination pattern is independent of the detection objective lens in chip-based ESI, which is in contrast to illumination being provided through the objective lens as performed by Kim et al. Presumably, the signal to noise ratio and other factors also influence the obtainable resolution. Hence, it will be helpful to further investigate these effects in chip-based ESI which might also favour the optimization of the imaging process.

By theory, the lowest bound for possible fringe size resulting from mode interference is found for counterpropagating coherent waves which result in an intensity pattern in the form of a standing wave with half of the wavelength of the light wave. If the fringe size is expressed by a FWHM value, it is half of the wavelength of the wave forming the intensity pattern, i.e. one quarter of the wavelength of the interfering light. Related to the waveguide, the smallest possible value for the structure FWHM of the interference fringes is given by $\mathrm{FWHM}_{\min }=\lambda /\left(4 \mathrm{n}_{\text {eff }}\right)$, which results in $97 \mathrm{~nm}$ assuming a vacuum wavelength of $\lambda=660 \mathrm{~nm}$ and an effective refractive index of $\mathrm{n}_{\text {eff }}=1.7$ inside the waveguide.

To be able to compare to this theoretical value, we have measured the multi-mode interference pattern using dSTORM (Supplementary Fig. 11) and find fringe sizes that go down to $140 \mathrm{~nm}$, which is about $44 \%$ higher than the theoretically possible minimum fringe size. It is reasonable that the fringe size does not go down to the lowest possible value as 
the pattern results from the interference between multiple modes which are propagating in the same direction inside the waveguide.

We use the measured fringe sizes to model the image formation in waveguide-based ESI for emitter pairs at different distances. We simulate their subsequent illumination by sinusoidal patterns with random phases and random FHWM fringe sizes equally distributed between the experimentally obtained lowest value of $140 \mathrm{~nm}$ and an upper value of $420 \mathrm{~nm}$ (Methods). Analysing the data from 200 frames with ESI shows that two emitters spaced at the diffraction limited are easily resolved (Supplementary Fig. 10). This also applies for emitters spaced by $150 \mathrm{~nm}$ which is well below the diffraction limit while closer emitters at $125 \mathrm{~nm}$ spacing are not resolved. Again, we find a ratio between the illumination pattern from $660 \mathrm{~nm}$ vacuum wavelength and the achieved resolution of about 1.3.

As in case of waveguide based ESI, the interference pattern is generated within the waveguide and not by diffraction limited optics, the obtainable resolution enhancement is bigger when comparing to the diffraction limited resolution. Hence, we found a resolution of about $110 \mathrm{~nm}$ for Alexa 488 emitting at approx. $\lambda=523 \mathrm{~nm}$ while the diffraction limited resolution limit when employing a NA 1.2 lens is $218 \mathrm{~nm}$, and, thus, a resolution enhancement of about a factor of 2 . 


\section{Supplementary Note 2: Obtainable resolution for objective lenses of different NA in localization microscopy}

As it is rather uncommon to record data in SMLM with low NA objective lenses, we investigate the theoretical limit for the achievable localization precision and compare it to the experimentally obtained values in Supplementary Fig. 14.

Both the original model of Thompson et al. ${ }^{55}$ (here referred to as [a]) and its updated version by Mortensen et al. $^{56}$ (referred to as [b]) for the localization precision depending on the statistics of the captured image of an emitter are widely used. A slightly adapted version of the latter is also implemented in ThunderSTORM ${ }^{54}$ (here referred to as [c]), the software that we use for image reconstruction. Supplementary Fig. 14a shows the theoretical prediction for the localization precision using either a high NA 1.2 water immersion lens (solid line) or a low NA 0.4 air lens (dashed line) calculated from both models.

For calculations, we used the experimentally achieved parameters for Atto 488 of a mean count of 248 photons/localization and a mean standard deviation of the background of 3.9 photons for the NA 1.2 lens, and a mean count of 128 photons/localization and a mean standard deviation of the background of 4.7 photons for the NA 0.4 lens. Note that different photon count values might stem from different sources, e.g. a different single frame exposure time that was used for the measurements. The photon count values were extrapolated to other NA values by taking the light collection efficiency depending on the acceptance cone of the objective lens into account. Assuming an emission wavelength of $\lambda=523 \mathrm{~nm}$ for Atto 488, we calculate the standard deviation $\sigma$ of the fitted Gaussian-shaped point spread function (PSF) by assuming a FWHM equal to the Abbe resolution limit. The backprojected pixel widths $A$ were calculated by assuming a perfect match between the Nyquist sampling capacity of the camera and the resolution of the optical system, i.e. $A=\lambda / 2 \mathrm{NA} 1 / 2 \sqrt{2}$. Note that while this match is highly appreciated in diffraction-limited imaging, it is often not fulfilled for setups that only serve for SMLM imaging as adjusted backprojected pixel sizes may lead to better fitting results of single molecule emissions. However, this match is almost given for our setup: utilizing the NA 1.2 objective lens for Atto 488 imaging, we use a backprojected pixel width of $A=75.9 \mathrm{~nm}$, while the Nyquistoptimal value is $A=77.0 \mathrm{~nm}$. In case of NA 0.4 , the used backprojected pixel width is $A=228.3 \mathrm{~nm}$, while the Nyquist-optimal value is $A=231.1 \mathrm{~nm}$.

To compare to the theory, the experimentally achieved localization precisions are estimated in two different ways: 
- Using the statistics of the captured image of each emitter in the raw data, the reconstruction software [c] assigns to all localizations an estimated localization precision. The mean value over all localizations for NA 1.2 and for NA 0.4 is drawn in grey in Supplementary Fig. 14a, considering the standard deviation as error intervals.

- Another approach to estimating the experimentally achieved average localization precision is proposed by Endesfelder et al. ${ }^{32}$ (referred to as [d]). This method relies on computations on the distance to nearest neighbours of localizations in adjacent frames of the raw data as these possibly stem from the same molecule. Because this assumption is not always fulfilled, correction terms are considered in the computation. Using the set of localizations from the entire image stack, the findings for the localization precision are drawn in black in Supplementary Fig. 14a. Please note that this methods is based solely on the localization coordinates and that it ignores the statistics on the raw data, i.e. photon count, background etc.

Experimentally achieved localization precisions estimated by both methods and theoretical predictions nicely fit in case of the NA 1.2 lens. (For better clarity, the representation of the experimental results is slightly shifted in the direction of the abscissa, but both refer to the case of NA 1.2.)

A strong deviation becomes apparent in case of the NA 0.4 lens being used. It is self-evident that the statistics of the localization precision computed by the reconstruction software [c] match the theoretical predictions of the model of [b] as they are based on the same assumptions, while the broad variance is possibly due to high noise in the raw data, of which Supplementary Fig. 14c shows an example. For comparison, Supplementary Fig. 14d shows an example of the raw data for the NA 1.2 lens being used, and Supplementary Fig. $\mathbf{1 4 g}$ and Supplementary Fig. $\mathbf{1 4 h}$ show reconstruction of the same are for the entire image stack.

In contrast to the method of [c], the estimation by the method of [d] results in significantly higher values for the localization precision. To explain this, we performed experiments on a conventional inverted microscope configured for SMLM experiments (Supplementary Fig. 14b). As samples, we use $100 \mathrm{~nm}$ sized fluorescent beads in a low concentration. This approach allows for modelling of single molecule localization experiments with adjustable image statistics comparable to the experiments on the waveguide (e.g. photon count values) by appropriately choosing excitation power and exposure times. Examples from the raw data are shown in Supplementary Fig. 14e and Supplementary Fig. 14f for the used objective lenses of NA 0.35 and NA 1.49, respectively. 
As the beads do not blink, it was possible to localize them over a large number of frames ( $n=2046$ for NA 1.49 and $n=4153$ for NA 0.35 ), such that the localization precisions in $x-$ direction (red circle in Supplementary Fig. 14b) and y-direction (red diamond) can be measured directly as the standard deviations of the localization distribution, shown in Supplementary Fig. 14i and Supplementary Fig. 14j. The comparison to the estimation by both methods shows good agreement with the directly measured values for both high and low NA lenses utilized. The higher value for the localization precision in $\mathrm{x}$-direction for the NA 0.35 case is possibly due to drift on the order of $10 \mathrm{~nm}$ during the experiment. Again, the localization precision values computed by reconstruction software show a broad variance, which is consistent with the findings on the waveguide.

Inconsistently, we find that in case of the low NA for the bead experiment (Supplementary Fig. 14b), the deviation between the estimation of the methods of [c] and [d] is not given as in case of the waveguide experiment (Supplementary Fig. 14a). Taking the raw data in case of the low NA lens (Supplementary Fig. 14c) and the high NA lens (Supplementary Fig. 14d) on the waveguide into account shows that the emitter density looks comparable. However, the individual PSFs are much worse separated in case of the low NA simply due to the significantly larger PSF widths. As the locations of the blinking dyes in a dSTORM experiment are distributed stochastically, wider PSFs increase the chances of overlapping PSFs (Supplementary Note 3) which can lead to (1) a wrong emitter position estimation as a localization will be found in between the real positions of the multiple emitters, and (2) a wrong estimation on the single emitter photon count as this will be based on the photon emission of the multiple emitters simultaneously. This can now explain the deviation of the localization precision estimations by the methods of [c] and [d] considering their underlying principles: The method of [c] performs an estimation of the precision for each localization on the statistics of the raw data independent of the complete reconstructed image. If an erroneous localization occurs due to overlapping PSFs of multiple emitters, this affects the quality of the reconstructed image but also the localization precision statistics. In contrast, [d] performs computations only on the localization coordinate list that is used to render the super-resolved image. Hence, deviations between the two methods can indicate the problem of too dense single molecule blinking in the raw data.

However, due the stochastic nature of the process, overlapping of the PSFs of multiple

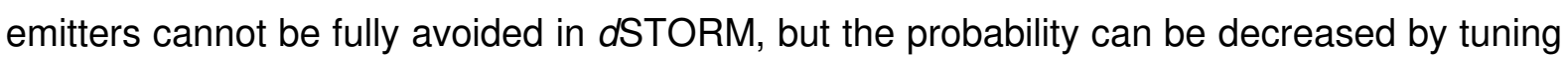
the experimental conditions in terms of imaging buffer composition, excitation power, single frame exposure time, and not least choosing an appropriate dye. While Atto 488 performs well for multiple subsequent acquisitions of the same sample that we used to compare between different modalities (Fig. 3) due to its relative good photostability ${ }^{60}$, Alexa 647 is 
often considered as a better option if the focus is laid on dSTORM imaging only. Consequently, we could achieve a higher resolution in dSTORM imaging using the latter dye (Fig. 2). This is confirmed by the very good agreement for the localization precisions determined by both methods for low and high NA chip-based dSTORM imaging (Supplementary Fig. 1 and Supplementary Fig. 6). Anyway, it was possible to achieve a resolution beyond the diffraction limit for low NA $(0.4$ and 0.45$)$ lenses on the waveguide for both Atto 488 and Alexa 647, showing that our approach allows for large FOV dSTORM imaging in TIRF excitation. 


\section{Supplementary Note 3: Resolution estimation on emitter density}

A condition for the lower bound on the achievable resolution referring to the density of simultaneously active emitters, i.e. the rate of blinking, was introduced by $\mathrm{Xu}$ et al. $^{13}$ and given as $\left(\pi(\lambda /(2 N A))^{2} r_{\max }\right)^{1 / 2}$. As the parameters of the equation, we assume the emission wavelength $\lambda=523 \mathrm{~nm}$ (estimated here as the emission spectrum peak of Atto 488), the numerical aperture of the setup $N A=0.4$, and the ratio between the maximum number of detected localization in one frame divided by the overall number of localization $r_{\max }=1,821 / 9,628,118$. This estimation gives a lower bound of $16 \mathrm{~nm}$ which is significantly lower than the actually achieved resolution, so it should not set a limit in this case.

However, this assumption takes dense packing of emitters without overlap of their PSFs into account. As the locations of active emitters are independent of each other and randomly distributed, dense packing without overlap is unlikely to occur in a dSTORM experiment, but overlap of active emitters by their PSF will occur at a certain probability depending on the labelling density, photoswitching properties, and PSF size ${ }^{61}$. Assuming the size of a diffraction limited spot as $D=\pi(\lambda /(2 \mathrm{NA}))^{2}$ similar to the estimation by $\mathrm{Xu}$ et al. ${ }^{13}$ and the overall imaged area $A$, the probability for one specific emitter to overlap with a second emitter is given by D/A. Hence the probability for no overlap with a second emitter follows as 1-D/A and for no overlap with $m$ other emitters as $(1-D / A)^{m}$. Consequently, the probability for one specific emitter to not overlap with any other emitter in case of $n=m+1$ simultaneously active emitters if given by $1-(1-D / A)^{n-1}$. For the dSTORM image shown in Fig. 4c, we have $A=3,456 \mu m^{2}, \lambda=523 \mathrm{~nm}, \mathrm{NA}=0.4$. The maximum number of simultaneously active emitters is $n_{\max }=1,821$ while the mean number of simultaneously active emitters is $n_{\text {mean }}=959$ (Supplementary Note 4). It follows that for $n_{\max }$, each emitter has the probability to overlap with another emitter of $50,7 \%$, while for $n_{\text {mean }}$, the probability is $31,1 \%$. Overlapping PSFs will lead to false localizations and can therefore affect the quality of the reconstructed image (Supplementary Note 2), also depending on the ability of the reconstruction algorithm to discern close emitters. 


\section{Supplementary Note 4: Resolution estimation on localization density}

As pointed out in ${ }^{62,63}$, the Nyquist resolution limit can be defined as $2 /(\text { localization density })^{1 / 2}$ in the case of two-dimensional localization microscopy, e.g. dSTORM. Accordingly, to allow for a Nyquist resolution on the order of $140 \mathrm{~nm}$, the localization density has to exceed 205 localizations $/ \mu \mathrm{m}^{2}$. However, as has been discussed recently ${ }^{64}$, oversampling by at least a factor of 5 is desired due to the stochastic nature of localization microscopy. Hence, the localization density has to be 1020 localizations $/ \mu \mathrm{m}^{2}$ to support a resolution of $140 \mathrm{~nm}$. The low NA dSTORM reconstruction of Atto 488-phalloidin stained LSECs shown in Fig. 4c comprises of $9,628,118$ localizations in 10,044 frames over a field-of-view of $3,456 \mu^{2}$. Within this field-of-view, about $2 / 3$ of the area is actually covered by cells, densely packed with actin. From these numbers, we estimate the localization density in a reconstruction from 3,000 frames to be $9,628,118$ localizations $\times(3,000$ frames $) /(10,044$ frames $) \times(2 / 3 \times$ $\left.3,456 \mu \mathrm{m}^{2}\right)^{-1}=1,248$ localizations $/ \mu \mathrm{m}^{2}$. Thus, the achieved localization density exceeds 1020 localizations $/ \mu \mathrm{m}^{2}$ and gives a lower bound for the resolution on the order of $140 \mathrm{~nm}$. In case of Atto 488 imaging, we achieved a localization precision of $88 \mathrm{~nm}$ (Supplementary Fig. 16) indicating an achievable resolution on the order of $2 \times(2 \times \ln (2))^{1 / 2} \times$ localization precision $\approx 207 \mathrm{~nm}$. So the localization precision determines the limit for the resolution, but not the labelling density, which is further confirmed by the reconstructed image (Supplementary Fig. 15). 


\section{Supplementary Note 5: Comparison to large FOV dSTORM via mosaic stitching}

Fig. 2e shows dSTORM imaging at approx. $140 \mathrm{~nm}$ resolution over a FOV of $0.5 \mathrm{~mm} \times 0.5 \mathrm{~mm}$ that has been reconstructed from 21,716 raw frames recorded in approximately 18 minutes (Supplementary Table 1). Circumventing the need for modifying the illumination scheme, the FOV for localization microscopy can also be extended by stitching of multiple areas as it has recently been demonstrated by Nahidiazar et al. ${ }^{65}$. The authors show that the stitching of eight regions resulted in the dSTORM image of an almost entire HUVEC cell. For each region, 20,000 raw frames at $100 \mathrm{~Hz}$ had been recorded on a commercial setup. Hence, 160,000 raw frames recorded in approx. 26 minutes and subsequent stitching were necessary for the dSTORM reconstruction of the entire cell. Though the authors do not give an estimated resolution for this example, we assume that the obtained resolution is much better than $140 \mathrm{~nm}$. However, using the waveguide-based approach and accepting a worse resolution (which is still well beyond the diffraction limit) more than 50 cells compared to 1 cell could be imaged in approx. 18 minutes compared to 26 minutes. 
Supplementary Table 1: Experimental conditions

\begin{tabular}{|c|c|c|c|c|c|c|c|c|c|c|}
\hline & Experiment & $\begin{array}{l}\text { Waveguide } \\
\text { material } \\
\text { and coupling } \\
\text { device }\end{array}$ & $\begin{array}{l}\text { Wave- } \\
\text { guided } \\
\text { breadth } \\
(\mu m)\end{array}$ & $\begin{array}{l}\text { Imaging } \\
\text { objective } \\
\text { lens }\end{array}$ & $\begin{array}{l}\text { Laser } \\
\text { vacuum } \\
\text { wavelength } \\
(\mathrm{nm})\end{array}$ & Dye & Approximate illumination intensity $\left(\mathrm{kW} / \mathrm{cm}^{2}\right)$ & $\begin{array}{l}\text { Exposure } \\
\text { time (ms) }\end{array}$ & \# frames & $\begin{array}{l}\text { Gaussian smoothing } \\
\text { kernel width (sigma) } \\
(\mathrm{nm})\end{array}$ \\
\hline Figure 2a & aSTORM & $\mathrm{Si}_{3} \mathrm{~N}_{4}$, fibre & 25 & 60x NA 1.2 & 660 & $\begin{array}{l}\text { Alexa } 647 \text { anti tubulin (primary and } \\
\text { secondary antibody staining) }\end{array}$ & 0.9 & 50 & 35043 & - \\
\hline Figure 2a & Diffraction limited & $\mathrm{Si}_{3} \mathrm{~N}_{4}$, fibre & 25 & 60x NA 1.2 & 660 & $\begin{array}{l}\text { Alexa } 647 \text { antit tubulin (primary and } \\
\text { secondary antibody staining) }\end{array}$ & 0.05 & 100 & 505 & - \\
\hline Figure $2 c$ & aSTORM & $\mathrm{Ta}_{2} \mathrm{O}_{5}$ objective & 65 & $60 \times \mathrm{NA} 1.2$ & 660 & Alexa 647 & 0.1 & 30 & 24665 & 10 \\
\hline Figure $2 c$ & aSTORM & - & - & 60x NA 1.49 & 647 & Alexa 647 & 5 & 50 & 6026 & 10 \\
\hline Figure 2e,f & aSTORM & $\mathrm{Si}_{3} \mathrm{~N}_{4}$, objective & 500 & 20x NA 0.45 & 660 & $\begin{array}{l}\text { Alexa } 647 \text { antit tubulin (primary and } \\
\text { secondary antibody staining) }\end{array}$ & 0.9 & 50 & 21716 & - \\
\hline Figure 2e,f & Diffraction limited & $\mathrm{Si}_{3} \mathrm{~N}_{\mathrm{t}}$ objective & 500 & 20x NA 0.45 & 660 & $\begin{array}{l}\text { Alexa } 647 \text { antit tubulin (primary and } \\
\text { secondary antibody staining) }\end{array}$ & 0.0004 & 100 & 525 & - \\
\hline Figure 3b,c, Supplementary Figure 12b & Diffraction limited & $\mathrm{Ta}_{2} \mathrm{O}_{5}$ objective & 50 & 60x NA 1.2 & 488 & Alexa 488 anti alpha-tubulin & 0.003 & 300 & 202 & - \\
\hline Figure 3b,c, Supplementary Figure 12a,c & ESI & $\mathrm{Ta}_{2} \mathrm{O}_{5}$ objective & 50 & 60x NA 1.2 & 488 & Alexa 488 anti alpha-tubulin & 0.003 & 300 & 202 & - \\
\hline Figure $3 b, c$ & aSTORM & $\mathrm{Ta}_{2} \mathrm{O}_{\text {, objective }}$ & 50 & 60x NA 1.2 & 488 & Alexa 488 anti alpha-tubulin & 0.3 & 20 & 30041 & 16.3 \\
\hline Figure 4a,d Supplementary Figure 13b & Diffraction limited & $\mathrm{Ta}_{2} \mathrm{O}_{5}$ objective & 65 & 20x NA 0.4 & 488 & Atto 488 -phalloidin & 0.03 & 100 & 200 & \\
\hline Figure 4b,e, Supplementary Figure 13a,c & ESI & $\mathrm{Ta}_{2} \mathrm{O} \mathrm{s}$, objective & 65 & 20x NA 0.4 & 488 & Atto 488 -phalloidin & 0.03 & 100 & 208 & - \\
\hline Figure 4c,f, Supplementary Figure 15 & aSTORM & $\mathrm{Ta}_{2} \mathrm{O}_{\text {, objective }}$ & 65 & 20x NA 0.4 & 488 & Atto 488 -phalloidin & 0.4 & 160.84 & 10044 & 68.5 \\
\hline Figure $4 \mathrm{~g}$ & dSTORM & $\mathrm{Ta}_{2} \mathrm{O}_{5}$, objective & 65 & 60x NA 1.2 & 488 & Atto 488 -phalloidin & 0.4 & 40 & 15048 & 22.8 \\
\hline Figure 5a, Supplementary Figure $17 \mathrm{c}$ & aSTORM & $\mathrm{Ta}_{2} \mathrm{O}_{\text {; objective }}$ & 65 & 60x NA 1.42 & 488 & Atto 488 -phalloidin & 0.4 & 50 & 75018 & 30.4 \\
\hline Figure 5a, Supplementary Figure $17 \mathrm{c}$ & aSTORM & $\mathrm{Ta}_{2} \mathrm{O}_{\text {; objective }}$ & 65 & 60x NA 1.42 & 660 and 488 & CellMask Deep Red & $\begin{array}{l}\text { all frames: } 9 \mathrm{~kW} / \mathrm{cm}^{2} \text { for } 660 \mathrm{~nm} \text {; } \\
\text { frames } 7650 \text { to } 125565: 0.4 \mathrm{~kW} / \mathrm{cm}^{2} \text { for } 488 \mathrm{~nm}\end{array}$ & 70 & 125565 & 30.4 \\
\hline Supplementary Figure $3 a$ & Diffraction limited & - & - & 60x NA 1.49 & 647 & Alexa 647 & & 30 & 1 & - \\
\hline Supplementary Figure 3b & Diffraction limited & $\mathrm{Ta}_{2} \mathrm{O}_{\text {s objective }}$ & 42 & 60x NA 1.2 & 660 & Alexa 647 & 10 & 10 & 1 & - \\
\hline Supplementary Figure 5, Video 2 & Diffraction limited & $\mathrm{Ta}_{2} \mathrm{O}_{5}$, objective & 75 & 60x NA 1.2 & 660 & $200 \mathrm{~nm}$ TetraSpeck Microspheres & 6 & 100 & 229 (Video) & \\
\hline Supplementary figure $8 a$ & Diffraction limited & $\mathrm{Ta}_{2} \mathrm{O}_{5}$, objective & 42 & $60 \times$ NA1.2 & 660 & Alexa 647 & 0.01 & 100 & 200 & - \\
\hline Supplementary figure $8 d$ & Diffraction limited & - & - & 60x NA1.49 & 642 & Alexa 647 & & 100 & 200 & - \\
\hline Supplementary Figure 9a,b,c, Video 1 & Diffraction limited & $\mathrm{Ta}_{2} \mathrm{O}_{5}$ objective & 42 & 60x NA 1.42 & 660 & CellMask Deep Red & 2 & 100 & 61 (Video) & \\
\hline Supplementary Figure 9d, Video 1 & dSTORM & $\mathrm{Ta}_{2} \mathrm{O}_{5}$ objjective & 42 & 60x NA 1.42 & 660 & CellMask Deep Red & 13 & 100 & 416770 & 30.4 \\
\hline Supplementary Figure 11 & aSTORM & $\mathrm{Ta}_{2} \mathrm{O}_{\text {, objective }}$ & 42 & 60x NA 1.2 & 660 & Alexa 647 & 11 & 10 & & \\
\hline Supplementary Figure 14e & Emitter detection & - & - & $20 x$, NA 0.35 & 647 & $200 \mathrm{~nm}$ TetraSpeck Microspheres & 0.03 & 15.37 & 2052 & - \\
\hline Supplementary Figure $14 f$ & Emitter detection & - & - & 60x NA 1.49 & 647 & $200 \mathrm{~nm}$ TetraSpeck Microspheres & 0.01 & 5 & 4153 & \\
\hline Supplementary Figure 17a & Diffraction limited & $\mathrm{Ta}_{2} \mathrm{O}_{5}$, objective & 65 & 60x NA 1.42 & 488 & Atto 488 -phalloidin & 0.03 & 200 & 200 & - \\
\hline Supplementary Figure 17b & Diffraction limited & $\mathrm{Ta}_{2} \mathrm{O}_{5}$ objective & 65 & 60x NA 1.42 & 660 & CellMask Deep Red & 5 & 100 & 300 & - \\
\hline
\end{tabular}




\section{REFERENCES FOR SUPPLEMENTARY INFORMATION}

13 Xu, J. Q., Tehrani, K. F. \& Kner, P. Multicolor 3D Super-resolution Imaging by Quantum Dot Stochastic Optical Reconstruction Microscopy. Acs Nano 9, 2917-2925, doi:10.1021/nn506952g (2015).

32 Endesfelder, U., Malkusch, S., Fricke, F. \& Heilemann, M. A simple method to estimate the average localization precision of a single-molecule localization microscopy experiment. Histochemistry and cell biology 141, 629-638 (2014).

33 Nieuwenhuizen, R. P. et al. Measuring image resolution in optical nanoscopy. Nat Methods 10, 557-562, doi:10.1038/nmeth.2448 (2013).

34 Banterle, N., Bui, K. H., Lemke, E. A. \& Beck, M. Fourier Ring Correlation as a resolution criterion fer super resolution microscopy. Journal of Structural Biology 183 (2013).

35 Douglass, K. M., Sieben, C., Archetti, A., Lambert, A. \& Manley, S. Super-resolution imaging of multiple cells by optimised flat-field epi-illumination. Nat Photonics 10, 705708, doi:10.1038/nphoton.2016.200 (2016).

39 Kim, M., Park, C., Rodriguez, C., Park, Y. \& Cho, Y. H. Superresolution imaging with optical fluctuation using speckle patterns illumination. Sci Rep-Uk 5, 16525, doi:10.1038/srep16525 (2015).

54 Ovesny, M., Krizek, P., Borkovec, J., Svindrych, Z. \& Hagen, G. M. ThunderSTORM: a comprehensive ImageJ plug-in for PALM and STORM data analysis and superresolution imaging. Bioinformatics 30, 2389-2390, doi:10.1093/bioinformatics/btu202 (2014).

55 Thompson, R. E., Larson, D. R. \& Webb, W. W. Precise nanometer localization analysis for individual fluorescent probes. Biophys $J$ 82, 2775-2783, doi:10.1016/S0006-3495(02)75618-X (2002).

56 Mortensen, K. I., Churchman, L. S., Spudich, J. A. \& Flyvbjerg, H. Optimized localization analysis for single-molecule tracking and super-resolution microscopy. Nat Methods 7, 377-381, doi:10.1038/nmeth.1447 (2010).

57 Mlodzianoski, M. J. et al. Sample drift correction in 3D fluorescence photoactivation localization microscopy. Opt Express 19, 15009-15019, doi:10.1364/OE.19.015009 (2011).

58 Mattheyses, A. L. \& Axelrod, D. Direct measurement of the evanescent field profile produced by objective-based total internal reflection fluorescence. Journal of biomedical optics 11, 014006-014006-014007 (2006).

59 Stabley, D. R., Oh, T., Simon, S. M., Mattheyses, A. L. \& Salaita, K. Real-time fluorescence imaging with $20 \mathrm{~nm}$ axial resolution. Nature communications 6 (2015).

60 Dempsey, G. T., Vaughan, J. C., Chen, K. H., Bates, M. \& Zhuang, X. Evaluation of fluorophores for optimal performance in localization-based super-resolution imaging. Nat Methods 8, 1027-1036, doi:10.1038/nmeth.1768 (2011).

61 van de Linde, S., Wolter, S., Heilemann, M. \& Sauer, M. The effect of photoswitching kinetics and labeling densities on super-resolution fluorescence imaging. J Biotechnol 149, 260-266, doi:10.1016/j.jbiotec.2010.02.010 (2010).

62 Shroff, H., Galbraith, C. G., Galbraith, J. A. \& Betzig, E. Live-cell photoactivated localization microscopy of nanoscale adhesion dynamics. Nature Methods 5, 417423, doi:10.1038/Nmeth.1202 (2008).

63 Shim, S. H. et al. Super-resolution fluorescence imaging of organelles in live cells with photoswitchable membrane probes. P Natl Acad Sci USA 109, 13978-13983, doi:10.1073/pnas.1201882109 (2012).

64 Legant, W. R. et al. High-density three-dimensional localization microscopy across large volumes. Nat Methods, doi:10.1038/nmeth.3797 (2016).

65 Nahidiazar, L., Agronskaia, A. V., Broertjes, J., van den Broek, B. \& Jalink, K. Optimizing Imaging Conditions for Demanding Multi-Color Super Resolution Localization Microscopy. Plos One 11, doi:10.1371/journal.pone.0158884 (2016). 